\title{
Interacciones humano-oso andino Tremarctos ornatus en el Perú: consumo de cultivos y depredación de ganado
}

\author{
Judith Figueroa ${ }^{1,2}$ \\ Asociación para la Investigación y Conservación de la Biodiversidad (AICB). Av. Vicús 538, Lima 33, Perú. E-mail: aicb.peru@gmail. \\ com \\ ${ }^{2}$ Grupo de Investigación de Zoología de Vertebrados. Universidad de Alicante. España.
}

\begin{abstract}
Introduction: The Andean bear Tremarctos ornatus has been hunted since the Inca times primarily because it was considered to be a pest that preyed on vicuñas and guanacos. Later, with the arrival of the Spaniards and the subsequent implementation of cattle ranching bears exploited this new and abundant food source. This resulted in a strong negative attitude of people towards the bear as they were perceived to be preying upon cattle and they did not take into consideration the fact that bears may have just been scavenging on carcasses. In addition, the expansion of cornfields in the area resulted in more incidences of bears raiding crops. Reports of these negative interactions continue to date. This paper utilizes extensive surveys, based on interviews with farmers, in order to learn how, when and where these interactions occur, as well as to understand their perception about bears. Moreover, knowledge of these interactions in the mitigation of other ursid species and experiences in other countries are discussed.

Methods: Fifty communities in 12 regions of Peru, located in neighboring areas or buffer zones of 16 protected areas with records of the Andean bear were visited between 2002 and 2008 . Three hundred and ten personal interviews were conducted mainly with hunters, farmers and ranchers. A semi-estructured interview was carried out in which the following basic questions were queried: date, time, place and type of interaction (livestock or crop), record type (observation, traces), crop species eaten or species of preyed animal, event description (solitary or groups of bears entering the fields, ages of bears, etc.) and reason for killing the bear.
\end{abstract}

Results: Eighty six records of bear kills were obtained in 66 villages of the 16 protected areas that were evaluated. A large proportion of the people interviewed (60.8\%) said that they had killed a bear and $48.5 \%$ of them said that they killed the bear because they considered it to be detrimental to the crops and $42.2 \%$ of them considered them detrimental to the livestock. Twelve types of crops were reported to be consumed by bears and maize was the one most frequently consumed $(n=150,93.3 \%)$. In addition, five species of livestock were consumed and cattle (bovine) was preyed the most frequently $(n=131,95.9 \%)$. The frequency and types of attacks were highly variable, mostly conducted by solitary adult males bears. Thirteen people claimed to have seen a total of ten attacks, giving a detailed description, while three people commented that they only observed scavenging behavior.

Discussion and conclusions: Corn crop raiding by Andean bears is an opportunistic event, because it is a food with high levels of energy and protein, and crops are within easy access to premontane and montane forest and adjacent areas. Furthermore, although there are studies that confirm bear predation on cattle in Venezuela, Colombia, Ecuador and Bolivia, to date, studies on this had not been undertaken in Peru. However, 13 people provided very detailed information about their observations, which agreed with other studies in other countries, confirming that bear predation on cattle also occurs in Peru. Since both events are a strong motive to hunt Andean bears, it would be important to conduct research in order to determine the reasons why some bears prey on livestock and raid crops. Further work needs to be conducted in close collaboration with the local communities in order to raise appropriate mitigation measures taking into consideration the conditions of each place, in order to reduce human-bear conflicts and therefore diminish the hunting of this species.

Key words: Andean bear, conservation, consumption of crops, hunting, livestock depredation, mitigation, Peru. 


\section{Introducción}

La mayoría de las especies de osos son omnívoros oportunistas que pueden ser considerados plagas cuando son atraídos por los cultivos, y perjudiciales cuando atacan a los animales domésticos. El oso solar Helarctos malayanus, puede devastar plantaciones de coco, piña, papaya, caña de azúcar y palma aceitera (Normua et al. 2004; Fredriksson 2005). Los osos bezudos Melursus ursinus, dañan sembríos de caña de azúcar y maní (Iswariah 1984 en Fredriksson 2005). El oso negro asiático Ursus thibetanus, ingresa a los cultivos de melocotón, ciruela, ciruela pasa, pera, papaya, maíz, plátano, mijo italiano y camote (Hwang et al. 2002) y a granjas de peces (Huygens y Hayashi 1999). El oso pardo U. arctos y el oso negro americano U. americanus, se alimentan de los apiarios, cultivos de maíz, avena y frutales, así como de ovejas, cabras, caballos, vacunos y cerdos (Jonker et al. 1998; Garshelis et al. 1999; Vargas y Hernández 2001).

En el caso del oso andino Tremarctos ornatus, este fue considerado como un animal dañino durante la época incaica $(1,438$ - 1,532) junto con el puma Puma concolor, por ser depredadores del "ganado silvestre" como vicuñas Vicugna vicugna, guanacos Lama guanicoe, tarucas Hippocamelus antisensis y venados Odocoileus virginianus, por lo que fueron capturados en la "cacería real del Inca", conocida como chaco (Garcilaso de la Vega 1609). Posteriormente, con la llegada de los españoles, los valles costeños, sierras y montañas se poblaron del ganado traído por ellos (bovino, ovino, caprino, equino, mular y porcino). Esto proporcionó una nueva y abundante fuente de alimento para los osos, principalmente de ganado vacuno, lo que conllevó a una mayor interacción con los humanos (Ponce 1991 [siglo XVI]; Jiménez de la Espada 1965 [1582]; Lequanda 1793b; Acosta 1849; Tschudi 2003 [1838-1842]), quienes no diferenciaron si se trataba de un evento predatorio o carroñero. Asimismo, los españoles extendieron los cultivos de maíz y frutales en algunas regiones (Rostworowski 1981), por lo que los osos también aprovecharon de estos (Lequanda 1793a; Tschudi 2003 [1838-1842]). Estas interacciones se presentan hasta la actualidad tanto en el Perú (Peyton 1980, 1983; Figueroa y Stucchi 2002; Baiker 2011; Figueroa et al. 2013) como en Venezuela, Colombia, Ecuador y Bolivia (Goldstein et al. 2006).

A diferencia del consumo de los cultivos por parte de los úrsidos de forma extendida, el ataque al ganado solo se realiza por algunos individuos (Jorgensen 1979; Wick 1995; Castellanos et al. 2011; Laguna 2013), cuyas causas exactas aún se desconocen. Sin embargo, debido a la percepción de que todos los osos son depredadores, los eventos de interacción oso-ganado provocan la cacería del oso "infractor" y de osos "inocentes" de la zona (Goldstein 2002; Achig 2009; Castellanos et al. 2011).

La depredación del ganado y el consumo de los cultivos por parte del oso andino, influyen directamente en la economía de los campesinos y la conservación de la especie, la cual aún no se ha analizado a profundidad. Si bien los resultados que se presentan a continuación se basan en entrevistas, que en algunos casos podrían estar sesgadas por el temor de los campesinos a dar información sobre la cacería ilegal de una especie, estas nos brindan su percepción y un alcance sobre cómo, cuándo y dónde se producen estas interacciones en el Perú. Esta recopilación podrá servir como una herramienta de trabajo inicial para establecer propuestas de mitigación acorde con las condiciones de cada localidad.

\section{Materiales y métodos}

Área de estudio. Entre los años 2002 y 2008 se visitaron 50 comunidades de 12 regiones del Perú, ubicadas en las zonas de amortiguamiento o aledañas a 16 áreas protegidas con registros de presencia del oso andino (Figura 1, Tabla 1). 
Evaluación. Se realizaron 310 entrevistas personales, principalmente a cazadores, agricultores y ganaderos, de edad adulta y con mucha experiencia en el campo. Se aplicó una entrevista semiestructurada, en la que se mantuvo una conversación fluida, basada en una lista de preguntas desarrollada previamente. Estas fueron: fecha, hora, lugar y tipo de interacción (ganado o cultivos), tipo de registro (observación, rastros), especie de cultivo consumido o animal depredado, descripción del evento (ingresaron osos solitarios o en grupos, edades de los osos, etc.), motivo de la cacería. Se visitaron algunas áreas donde se reportaron la depredación del ganado y el consumo de cultivos para observar las características del lugar.

\section{Resultados}

En todas las zonas de amortiguamiento de las áreas protegidas evaluadas se observó una ganadería extensiva y cultivos en áreas rodeadas por bosques montanos y premontanos, o limitados por estos, muy distantes a una vivienda (Figura 2a, 2b). Se visitaron 32 áreas donde ocurrieron eventos de interacción oso-ganado y oso-cultivo de maíz. De los 19 cultivos observados, 15 (78.9\%) se encontraron colindantes al bosque, mientras que cuatro (21.1\%) estuvieron a menos de $1 \mathrm{~km}$ de una carretera o caserío. Las 13 áreas donde se produjeron interacciones con el ganado se encontraron colindantes al bosque; en estas, se observó una escasa supervisión, el ganado era visitado solo una vez cada 20 o 30 días, sin ningún control de las hembras preñadas ni de los individuos jóvenes.

Se obtuvieron 86 registros de la cacería del oso en 66 poblados localizados en las zonas de amortiguamiento de las 16 áreas protegidas evaluadas (Figura 3a, 3b, 3c, Tabla 2). El $60.8 \%$ ( $n=$ 310 ) de los entrevistados señalaron que cazan al oso andino, y que esta se realiza principalmente por ser considerado un animal perjudial ya que se alimenta de los cultivos (48.5 \%) y ataca al ganado (42.2 \%) (Tabla 3). El $7.0 \%$ señaló que lo cazan para su uso alimenticio, y un $2.3 \%$ para la obtención de oseznos como mascotas. El reporte más antiguo de cacería fue en 1953, continuando hasta el mismo año en que se dio la entrevista en cada una de las áreas visitadas. Los principales meses de caza fueron abril ( $n=19,31.6 \%)$ y mayo $(21.0 \%)$.

Consumo de cultivos (Figura 4a, 4b, 4c). Se reportaron 12 cultivos consumidos por el oso andino, donde el maíz fue señalado como el principal producto $(n=150,93.3 \%)$. Las demás especies reportadas fueron: caña de azúcar (1.6\%), palta/aguacate (1.4\%), tuna (1.3\%), zapallo macre $(0.5 \%)$, calabaza $(0.4 \%)$, chirimoya $(0.4 \%)$, zapallo loche $(0.3 \%)$, guayaba $(0.2 \%)$, lúcuma (0.2\%), papa (0.2\%) y piña (0.2\%; Tabla 4).

Existen reportes de hasta 10 individuos comiendo en un mismo cultivo de maíz en Yanachaga Chemillén (Cañachacra), de ocho en Machu Picchu y más de cuatro en Bahuaja Sonene (Sandia). De los individuos cazados por el ingreso a los campos de maíz $(n=40)$, el $35.9 \%$ fueron machos adultos, $17.9 \%$ hembras adultas, $15.4 \%$ hembras con crías, $23.1 \%$ oseznos y $7.7 \%$ jóvenes. Los ingresos se produjeron entre las 05:00 y 07:00 h y las 16:00 y 18:00 h. Estos se dieron entre diciembre y agosto, pero principalmente en marzo y abril $(17.0 \%, n=24)$, época en la que el maíz se encuentra tierno y jugoso (choclo).

En Tingo María y Yanachaga Chemillén se comentó que un oso podía llevarse diariamente entre 20 y 35 tallos de maíz a un lugar seguro para comerlos. Empieza a comer en la parte más alta, desde el borde del bosque con el maizal. Se reportó su preferencia por el maíz blanco, pero también puede alimentarse del maíz "pintado" si aún está en estado de choclo. Tres personas comentaron del consumo del maíz seco y almacenado en áreas adyacentes a Megantoni (cerca al Pongo de Mainique), Machu Picchu (San Miguel) y Huascarán, en donde también se encontraron restos vomitados por el oso.

Depredación de ganado (Figura 5a, 5b, 5c). La depredación al ganado vacuno fue la más frecuente ( $n=131,95.9 \%)$, seguida de lejos por el ataque a las cabras (1.6\%), ovejas (1.1\%), 


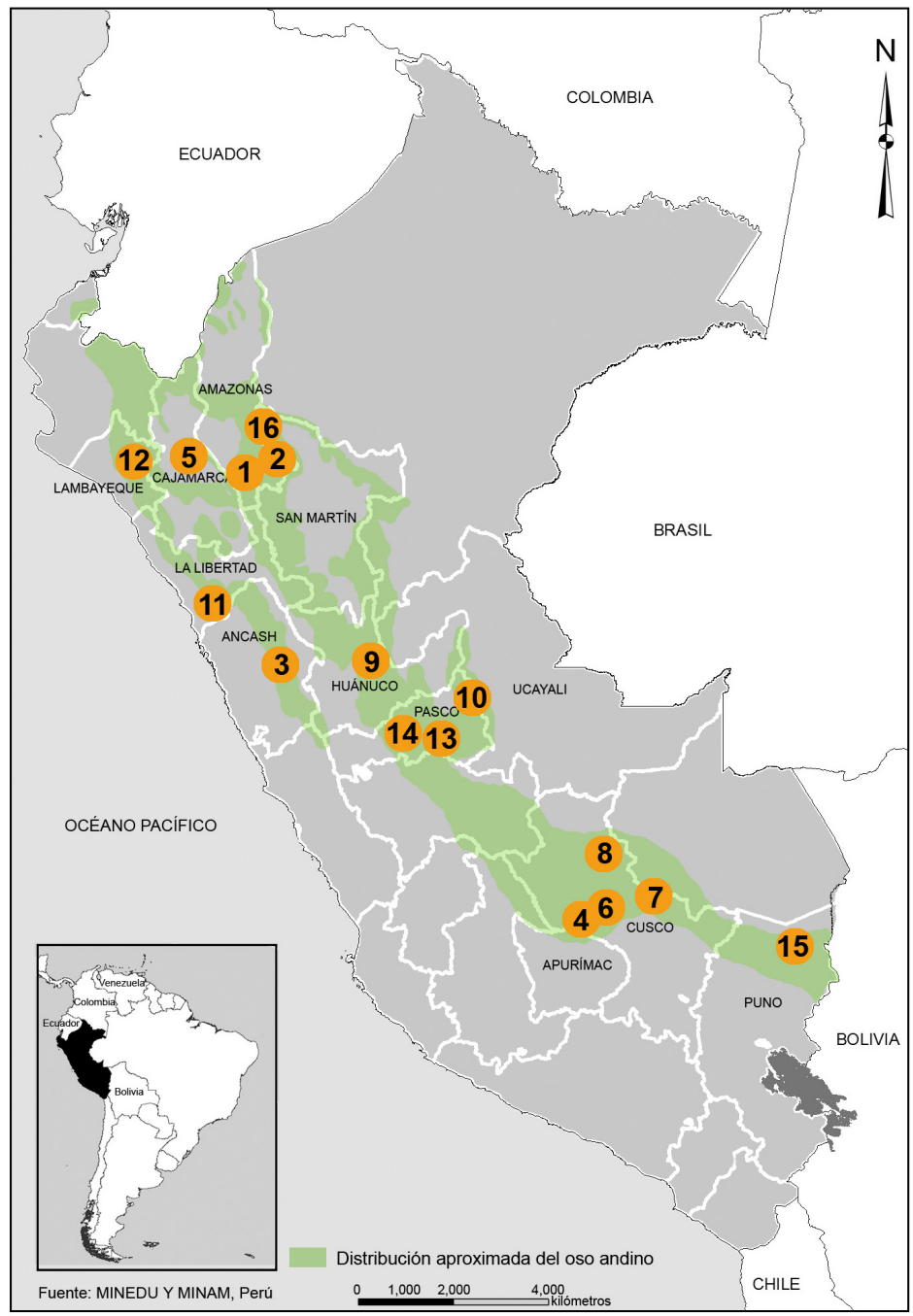

ÁREAS PROTEGIDAS DONDE SE REALIZARON ENTREVISTAS EN SUS ZONAS DE AMORTIGUAMIENTO 2002 - 2008

Área de Conservación Privada Huaylla Belén-Colcamar.

2 Área de Conservación Municipal Huamanpata.

3 Parque Nacional Huascarán.

4 Área de Conservación Regional Choquequirao.

5 Parque Nacional Cutervo.

6 Santuario Histórico Machu Picchu.

7 Parque Nacional Manu.

8 Santuario Nacional Megantoni.

9 Parque Nacional Tingo María.

10 Reserva Comunal EI Sira.

11 Reserva Nacional Calipuy.

12 Refugio de Vida Silvestre Laquipampa.

13 Bosque de Protección San Matías-San Carlos.

14 Parque Nacional Yanachaga Chemillén.

15 Parque Nacional Bahuaja Sonene.

16 Bosque de Protección Alto Mayo.

Figura 1. Áreas protegidas donde se realizaron las entrevistas en sus zonas de amortiguamiento o aledañas.

cerdos (0.7 \%) y caballos (0.7 \%) (Tabla 5). De las 310 personas entrevistadas, 13 aseguraron haber presenciado un total de diez eventos, dando una descripción detallada. Estos eventos depredatorios se produjeron principalmente en abril y mayo $(n=11,36.4 \%)$, entre las 19:00 $\mathrm{h}$ y 04:00 h. Se obtuvieron varios reportes de estos ataques en diferentes periodos: en Cutervo, Laquipampa y Tingo María cesaron en 1982, 1990 y 1993, respectivamente. En Megantoni, Huaylla Belén-Colcamar, Huamanpata, Machu Picchu, Manu, Yanachaga Chemillén y Alto Mayo se obtuvieron reportes hasta el mismo año en que se realizaron las entrevistas.

De igual manera, las frecuencias de ataques fueron bastante variables. En Choquequirao (Lucmabamba) un oso macho atacó cinco vacas en un mes. En Yanachaga Chemillén (Huaylas y Santa Bárbara), en la década de 1990 el oso podía atacar entre una y tres vacas al año. En Bahuaja Sonene (Iparo), en el 2005, un oso macho atacó y se alimentó de 20 vacas. Al cazarlo, se observó que sus dientes estaban muy desgastados. Por ello, la persona entrevistada aseguró que los osos que atacan al ganado vacuno, son los viejos que no pueden desplazarse largas distancias en busca 
Tabla 1. Comunidades entrevistadas entre el 2002 y 2008.

\begin{tabular}{|c|c|c|c|c|}
\hline $\begin{array}{l}\text { Zona de amortiguamiento o } \\
\text { aledaña - Área protegida }\end{array}$ & Región & Localidades visitadas & Año & Entrevistados \\
\hline ACP Huaylla Belén-Colcamar & Amazonas & Luya, Leimebamba y La Jalca & 2002 & 16 \\
\hline ACM Huamanpata & Amazonas & Rodríguez de Mendoza y Huambo & 2002 & 6 \\
\hline PN Huascarán & Ancash & Ecash y Colcas & 2002 & 17 \\
\hline \multirow{2}{*}{ ACR Choquequirao } & Apurímac & Huanipaca, Cachora y Abancay & 2002 & 9 \\
\hline & Cusco & Lucmabamba & 2002 & 7 \\
\hline PN Cutervo & Cajamarca & $\begin{array}{l}\text { Paraíso, Pucarilla, San Andrés de } \\
\text { Cutervo, La Capilla y Santo Tomás }\end{array}$ & 2002,2003 & 31 \\
\hline SH Machu Picchu & Cusco & Santa Teresa y Mándor & 2002,2003 & 26 \\
\hline PN Manu & Cusco & Challabamba y Paucartambo & 2003 & 11 \\
\hline SN Megantoni & Cusco & $\begin{array}{l}\text { Timpía, Matoriato y Shivankoreni, } \\
\text { Lacco, Yanacocha y Lorohuachana }\end{array}$ & 2004,2008 & 15 \\
\hline PN Tingo María & Huánuco & Juan Santos Atahualpa & 2002 & 19 \\
\hline \multirow{2}{*}{ RC El Sira } & Huánuco & $\begin{array}{l}\text { Puerto Inca, Zúngaro, El Sira y } \\
\text { Yuyapichis }\end{array}$ & \multirow{2}{*}{2002} & \multirow{2}{*}{28} \\
\hline & Ucayali & Atalaya, Padre Abad y Aguaytía & & \\
\hline RN Calipuy & La Libertad & Calipuy y Paibal & 2002 & 8 \\
\hline RVS Laquipampa & Lambayeque & Mochumí, Higuerón y Laquipampa & $\begin{array}{c}2002,2003, \\
2004\end{array}$ & 25 \\
\hline BP San Matías-San Carlos & Pasco & Iscozasín y San Francisco & 2003,2007 & 8 \\
\hline PN Yanachaga Chemillén & Pasco & $\begin{array}{l}\text { Santa Bárbara, Huayruro, Cañachacra, } \\
\text { Mal Paso, Pozuzo y Yulitunqui }\end{array}$ & $\begin{array}{c}2003,2005, \\
2007\end{array}$ & 54 \\
\hline PN Bahuaja Sonene & Puno & Sandia & 2006 & 7 \\
\hline BP Alto Mayo & San Martín & Alto Mayo y Nueva Cajamarca & 2002 & 23 \\
\hline
\end{tabular}

ACM - Área de Conservación Municipal, ACP - Área de Conservación Privada, ACR - Área de Conservación Regional, BP - Bosque de Protección, PN - Parque Nacional, RVS - Refugio de Vida Silvestre, RC - Reserva Comunal, RN - Reserva Nacional, SH - Santuario Histórico, SN - Santuario Nacional.

de su alimento natural. Señalaron que el oso tiende a atacar al ganado en un sector por un periodo de tiempo y luego migra hacia otra área.

El $67.7 \%(n=31)$ de los osos que atacaron al ganado fue cazado, ya que los campesinos siguieron los rastros dejados por estos al jalar sus presas para alimentarse en otras zonas. El $83.9 \%$ fueron machos adultos solitarios, solo en cinco ocasiones (16.1\%) se informó que las hembras tuvieron este comportamiento: dos hembras adultas solitarias, una hembra con un macho de pareja y dos hembras con oseznos. En Manu (Sunchubamba), en 1999, una osa mató una vaca, la que comió durante tres días. En Calipuy (Paibal), en el 2001 una osa mató a 15 terneros en menos de un mes. Otra desbarrancó a un toro de $300 \mathrm{~kg}$, del cual se alimentaron ella y sus dos oseznos por cinco días. De la misma manera, se reportó en Cutervo (La Capilla) este comportamiento de una hembra con dos crías en 1982. En Machu Picchu (Chachabamba), en 1999, un campesino presenció a una pareja 

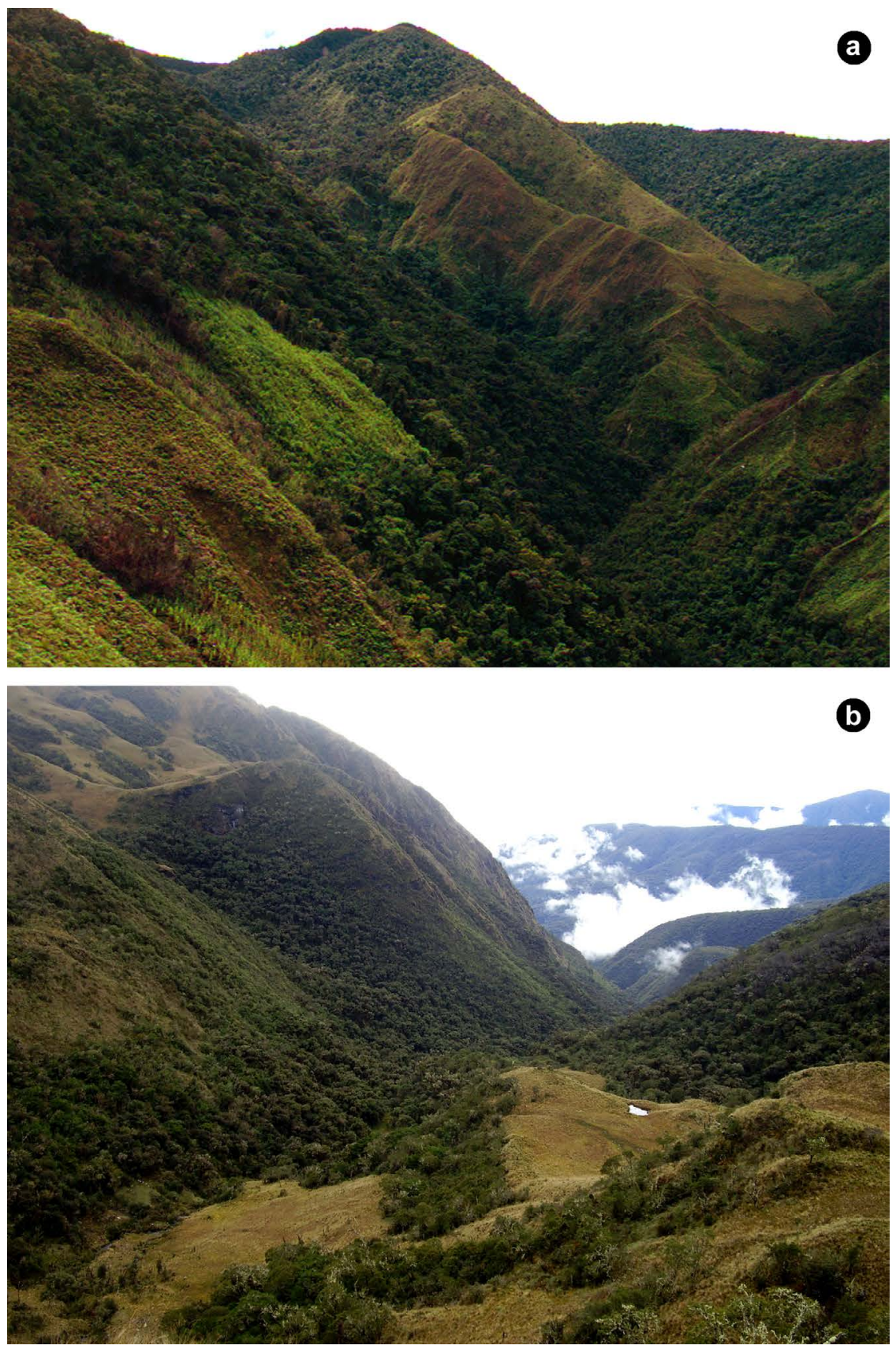

Figura 2. Áreas con reportes de interacción humano-oso andino. a) Cañachacra (Pasco), cultivos de maíz rodeados de bosque. b) Lorohuachana (zona de amortiguamiento de Megantoni), área ganadera limitando con el bosque.

de osos corriendo detrás de un grupo de vacunos, concentrándose luego en un individuo al cual lograron desbarrancar; este fue comido en el mismo lugar donde cayó. Otro caso similar se dio en el 2000 en Choquequirao (Rajche) con un oso solitario. En Calipuy, tres pobladores que observaron el hecho, comentaron que seis osos dirigieron a las vacas cerca al precipicio, luego a una de ellas un oso "le agarró y giró la cola" para que esta caiga con su propio peso. En Laquipampa (El Naranjo), en 1990, observaron que un oso se subió sobre el lomo de la vaca para morderla y quebrarle las escápulas con el fin de inmovilizarla. En la zona de amortiguamiento de Alto Mayo (hacia el lado de Amazonas) en el 2000, un poblador presenció el ataque de tres osos a una vaca; cerca a esta área, un año después un oso mató a un perro cuando este defendió el potrero. Algo similar ocurrió en Carpish (Huánuco), cuando un perro protegió un grupo de 
Tabla 2. Localidades donde se reporta el ingreso a los cultivos de maíz y ataque al ganado según las entrevistas.

\begin{tabular}{|c|c|c|}
\hline $\begin{array}{l}\text { Zona de amortiguamiento } \\
\text { o aledaña - Área protegida }\end{array}$ & Ingreso a cultivo de maíz & Ataque a ganado \\
\hline Huaylla Belén-Colcamar & San Carlos, Poblado Cortadera, Bongará & Quijalca, Leimebamba, Lajasbamba \\
\hline Huamanpata & & Huambo, Yamaniau \\
\hline Huascarán & & $\begin{array}{l}\text { Musho (Poquia), Waripata, Chacas, Quitaraxa, } \\
\text { Wicsonga }\end{array}$ \\
\hline Choquequirao & & Sacsara, Yanatile y Lucmabamba \\
\hline Cutervo & & $\begin{array}{l}\text { Gruta de los Guacharos, San Pedro, sector Capilla, } \\
\text { Playa Grande y Pucarilla }\end{array}$ \\
\hline Machu Picchu & Mándor & Santa Teresa, Chachabamba \\
\hline Manu & $\begin{array}{l}\text { Televán, Otocani, Pucará, El Rocotal y San Pedro, } \\
\text { cerca al río Yavero-Mapacho }\end{array}$ & $\begin{array}{l}\text { Challabamba, Trigomontón, Huaysampilla, El } \\
\text { Rocotal, Buenos Aires, Qurqurpampa y Nuevo } \\
\text { Oriente }\end{array}$ \\
\hline Megantoni & $\begin{array}{l}\text { Áreas adyacentes al río Yavero-Mapacho, al } \\
\text { Pongo de Mainique y Lacco }\end{array}$ & Yanacocha, Lorohuachana y Mishkiuno \\
\hline Tingo María & $\begin{array}{l}\text { Panao, Carpish, San Luis, Tambillo Grande y El } \\
\text { Mirador }\end{array}$ & \\
\hline El Sira & Santa Teresa y Cinchuna & \\
\hline Calipuy & & $\begin{array}{l}\text { Paibal, Quibal, Moyupuquio, Pichipata, Huaraday } \\
\text { y Tuntus }\end{array}$ \\
\hline Laquipampa & & El Naranjo, La Calera \\
\hline San Matías-San Carlos & $\begin{array}{l}\text { Áreas bajas de } 200 \mathrm{~m} \text { de altitud adycante a } \\
\text { Iscozasín }\end{array}$ & \\
\hline Yanachaga Chemillén & $\begin{array}{l}\text { Alto Palmapampa, Alto Lagarto Mallapampa, } \\
\text { Jushi, Osopampa, La Suiza, Choropampa, La } \\
\text { Rinconada, Cantarizú, Palmazú, Seso, Yulitunqui, } \\
\text { Delfín, Prusia, Chontabamba, Mal Paso, Río Seco } \\
\text { y Cañachacra }\end{array}$ & Santa Bárbara, Huaylas y Chinchango \\
\hline Bahuaja Sonene & $\begin{array}{l}\text { Sandia, Maucallajta, Acañiputo, Huancaluque, } \\
\text { Chichanaco, Mororía, Putina, Huancané y Chaco }\end{array}$ & Iparo \\
\hline Alto Mayo & No detallaron la localidad exacta & No detallaron la localidad exacta \\
\hline
\end{tabular}

ovejas. En Megantoni (Mishkiuno), se observó el cráneo de un oso macho adulto, con las piezas dentarias muy desgastadas (Figura 5d), que fue cazado en el 2007 por atacar a un vacuno. Según nos informó el ganadero, después de cazar tres osos en la zona, la depredación concluyó.

Se observaron las características de los dientes de 14 cráneos de osos machos que fueron cazados, según nos comentaron, por depredar ganado vacuno, en todos los casos estos se encontraron muy desgastados o los caninos rotos. Los bovinos atacados ( $n=34$ ) fueron un $58.8 \%$ adultos, que incluyeron individuos de hasta siete años de edad (Manu, Nuevo Oriente) y hembras preñadas (Bahuaja Sonene, Sandia), y un $41.2 \%$ terneros.

Respecto a las partes de las cuales se alimenta el oso, en Manu (Qurqurpampa) un oso macho que mató a dos vacas, se alimentó de las ubres. En Laquipampa se reportó el ataque a vacas de 100 a 150 kg, de las que solo consumió la carne, dejando las vísceras. En Yanachaga Chemillén (Santa Bárbara), se reportó el ataque a vacunos, carneros y cerdos, de los que comió las vísceras y el hocico.

En tres casos el oso andino fue señalado como un animal carroñero. En Huascarán, en la laguna Parón en 1982, se observó a un oso jalando una vaca en estado de putrefacción para alimentarse de ella. En Cutervo, en el 2000, observaron a un oso macho jalando un ave en avanzado estado 

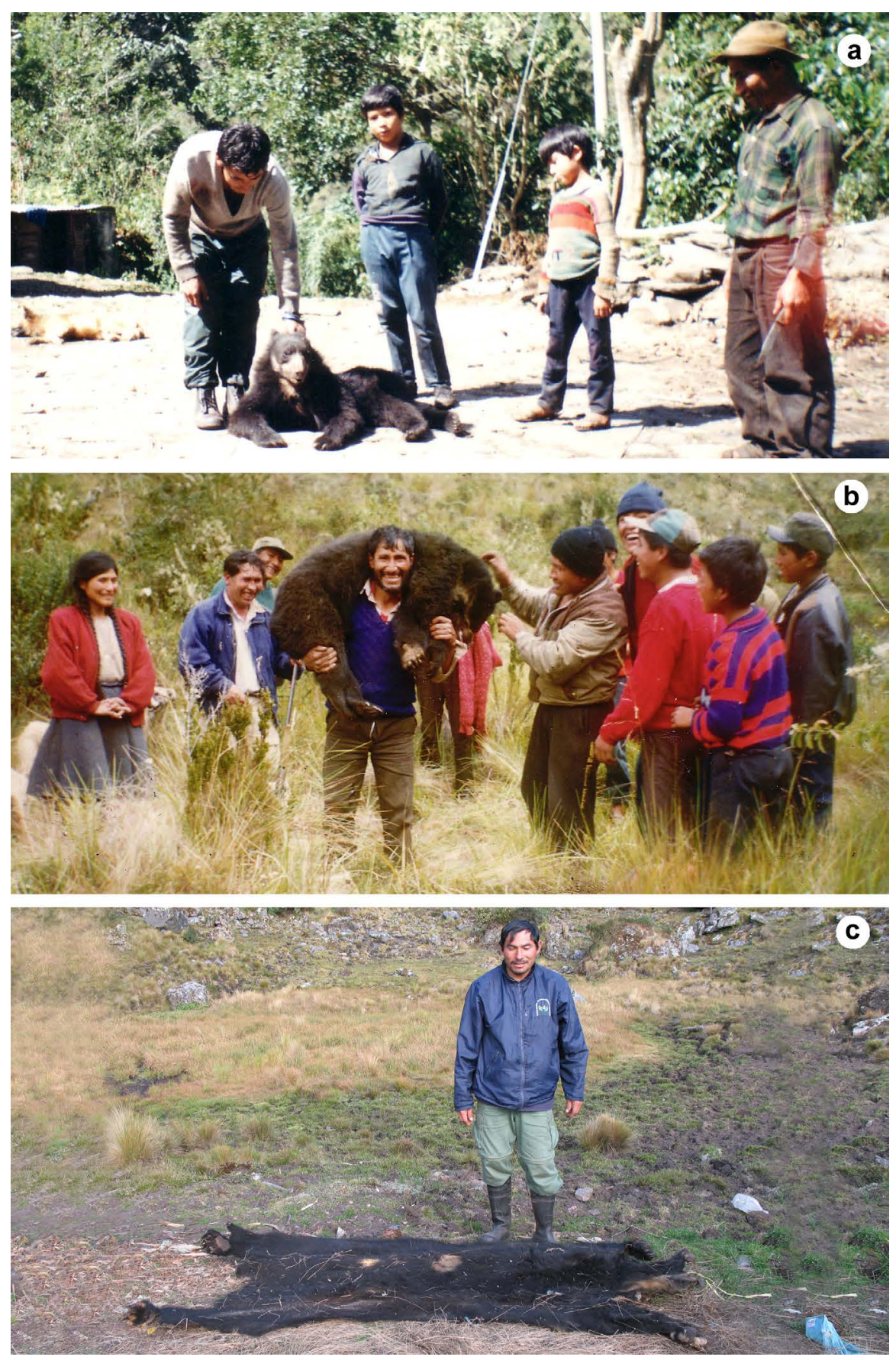

Figura 3. Cacería del oso andino por ingreso a los cultivos o depredación de ganado. a) Yanatile (zona de amortiguamiento de Machu Picchu), oso joven cazado en junio 1999 (Foto: Alan Valdeiglesias). b) Chinchalmainiyuj (zona de amortiguamiento de Manu), osa cazada en el 2001 (Foto: Milton Cabrera). c) Mishkiuno (zona de amortiguamiento de Megantoni), oso cazado en el 2007 por evento de depredación (Foto: Roberto Gutiérrez).

de descomposición en el borde de una laguna. En Calipuy, se observaron adultos y oseznos alimentándose de los restos de una vaca muerta en la orilla de un río.

Como consecuencia de la depredación de ganado, se manifestaron dos agresiones físicas de parte del oso a personas. En una oportunidad el oso rasguñó a un pastor que le tiró una piedra para que no ataque a sus ovejas en Huascarán (Ecash). En el año 2000, en Laquipampa (Rumichaca), un oso macho atacó a un campesino que arriaba a su ganado cuando intentó espantarlo. 
Tabla 3. Motivos de la cacería del oso andino según las entrevistas.

\begin{tabular}{|c|c|c|c|c|c|c|c|}
\hline \multirow{2}{*}{$\begin{array}{l}\text { Zona de amortiguamiento } \\
\text { o aledaña - Área protegida }\end{array}$} & \multirow{2}{*}{ Entrevistas } & \multicolumn{3}{|c|}{ ¿Cazan al oso andino? (\%) } & \multicolumn{3}{|c|}{$\begin{array}{l}\text { De los que respondieron “Sí, a la pregunta } \\
\text { anterior: ¿por qué cazaron al oso andino? (\%) }\end{array}$} \\
\hline & & Sí & No & No sabe & $\begin{array}{l}\text { Ingreso a } \\
\text { cultivos }\end{array}$ & Ataque al ganado & Otros* \\
\hline Huaylla Belén-Colcamar & 16 & 56.3 & 31.3 & 12.4 & 55.6 & 33.3 & 11.1 \\
\hline Huamanpata & 6 & 83.3 & 16.7 & & 60.0 & 20.0 & 20.0 \\
\hline Huascarán & 17 & 88.2 & 11.8 & & 6.7 & 93.3 & \\
\hline Choquequirao & 16 & 56.3 & 43.7 & & 88.9 & 11.1 & \\
\hline Cutervo & 31 & 9.7 & 87.1 & 3.2 & 66.7 & 28.0 & 5.3 \\
\hline Machu Picchu & 26 & 76.9 & 15.4 & 7.7 & 70.0 & 20.0 & 10.0 \\
\hline Manu & 11 & 90.9 & 9.1 & & 70.0 & 30.0 & \\
\hline Megantoni & 15 & 46.7 & 53.3 & & 14.3 & 85.7 & \\
\hline Tingo María & 19 & 21.1 & 73.7 & 5.2 & 100.0 & & \\
\hline El Sira & 28 & 10.7 & 89.3 & & 86.7 & 13.3 & \\
\hline Calipuy & 8 & 100.0 & & & & 100.0 & \\
\hline Laquipampa & 25 & 52.0 & 48.0 & & 7.7 & 92.3 & \\
\hline San Matias-San Carlos & 8 & 25.0 & 75.0 & & 20.0 & & 80.0 \\
\hline Yanachaga Chemillén & 54 & 70.4 & 24.1 & 5.5 & 71.1 & 23.7 & 5.2 \\
\hline Bahuaja Sonene & 7 & 85.7 & 14.3 & & 50.0 & 33.3 & 16.7 \\
\hline Alto Mayo & 23 & 100.0 & & & 8.7 & 91.3 & \\
\hline Total ( \%) & 310 & 60.8 & 37.0 & 2.2 & 48.5 & 42.2 & 9.3 \\
\hline
\end{tabular}

\section{Discusión}

La mayoría de las personas entrevistadas reconocen que la cacería del oso es un hecho ilícito, sin embargo, no consideran que atentan contra la ley cuando cazan a un oso para la defensa de sus cultivos o ganado, o como un acto para la obtención de una compensación por la pérdida: alimenticio, medicinal, venta de la piel o sus partes. En algunos casos, esta "defensa" es un pretexto para obtener un beneficio económico o como afición, como lo señalado por una persona en Quebrada Seca (Pasco), quien comentó que sembraba maíz cerca del bosque adrede para cazar al oso. Asimismo, en Challabamba (Cusco) un cazador señaló que era contratado por los campesinos perjudicados por el oso para ayudarlos con su problema. Las pieles y otras partes, productos de esta cacería, eran vendidas en los mercados de Cusco. El aseguraba que su trabajo era importante para la comunidad. Se pudo observar que tenía a la venta varias pieles de osos. Un reporte similar se dio en el norte del Perú, con un cazador que compensaba a los agricultores que le avisaban en qué maizal cazar al oso (Peyton 1980).

La cacería del oso, debido a las interacciones con el hombre se viene incrementando debido al crecimiento de la población y su invasión en las áreas boscosas (Figueroa et al. 2013). Desafortunadamente las mejores zonas para los cultivos y pastoreo coinciden con el mejor hábitat de producción natural de alimentos para el oso andino (Peyton et al. 1998).

En el presente estudio, los entrevistados señalaron que el consumo de los cultivos era el principal motivo de la cacería del oso, seguido del ataque al ganado. Resultados similares fueron encontrados en Colombia $(n=66)$, en el primer caso fue de $54.5 \%$ y el segundo de $39.4 \%$ (Jorgenson y Sandoval 2005). 

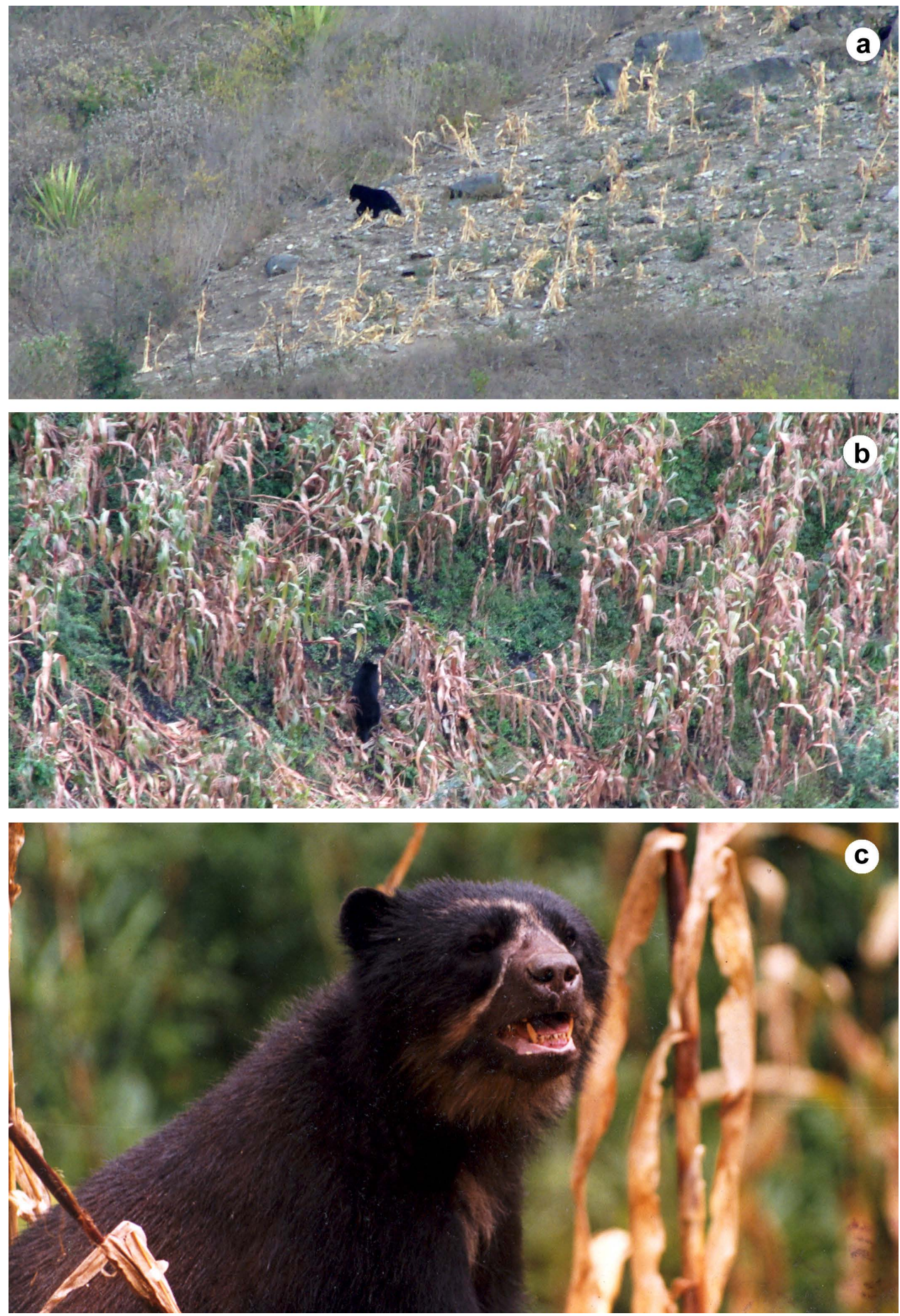

Figura 4. Ingreso del oso andino a los cultivos de maíz. a) Pilcomarca (Apurímac) (Foto: Jan Baiker/Ecobona-Cosude). b) Otocani (zona de amortiguamiento de Manu) (Foto: ProBosque Manu/Sociedad Zoológica de Frankfurt Perú). c) Puente San Pedro (zona de amortiguamiento de Manu; Foto: Oscar Mujica).

\section{Consumo de cultivos}

En las entrevistas se identificaron 12 cultivos consumidos por el oso, de los cuales maíz, palta/ aguacate, caña de azúcar, lúcuma y tuna ya habían sido registrados anteriormente por otros autores en Cusco y Apurímac, al sureste del Perú (Peyton 1980; Baiker 2011). Estos, junto con los siete nuevos registros en este trabajo (zapallo macre, zapallo loche, calabaza, chirimoya, guayaba, 
papa y piña), se suman a los de Chucén (Cajamarca) donde se reportó el consumo de plátano/banana y yuca/mandioca (Figueroa et al. 2013), y al de Quillabamba (Cusco) con la arracacha (Figueroa y Stucchi 2013), teniendo un total de 15 cultivos registrados en el Perú. Esto complementaría la recopilación de Figueroa (2013), teniendo un total de 18 cultivos reportados como consumidos por el oso andino en su área de distribución (Tabla 6). A excepción del maíz, estos cultivos representan recursos nutritivos de bajos niveles de energía, proteína y fibra en comparación con los frutos silvestres (Figueroa 2013), además, tomando en cuenta la baja frecuencia en estos ingresos, su ingesta sería un evento netamente oportunista. Por el contrario, en el caso particular del maíz, este presenta un alto nivel de energía y proteínas, siendo un alimento muy nutritivo (Dierenfeld 1989), que incluso, ha sido comido seco en algunos depósitos ubicados en la zona de amortiguamiento de Machu Picchu (Peyton 1983), así como en Megantoni y Huascarán. Esto explicaría su frecuente consumo en diversas áreas donde se distribuye. Esta se incrementa debido a que los campesinos siembran el maíz en áreas adyacentes a los bosques premontanos y montanos, donde habita naturalmente esta especie, y donde pueden alimentarse también de los frutos silvestres, bromelias y palmeras (Peyton 1980; Castellanos et al. 2005), esto se observó en el presente estudio y en otras áreas adyacentes al río Marañón (Figueroa et al. 2013). Sin embargo, también ingresan a campos de cultivo a menos de $1 \mathrm{~km}$ de la carretera, aunque de una forma menos frecuente; esto también fue observado por Leite Pitman (2008) en Cusco, a 2 km de la Carretera Interoceánica. Coincidiendo con Peyton (1980), los ingresos a los maizales ocurren principalmente cuando se encuentra como choclo. Los osos empiezan a alimentarse de esta planta entre los 1,500 a 2,000 msnm en febrero-marzo hasta los 2,700 msnm, en mayo-junio (Peyton 1987).

La cantidad de maíz comido por los osos fue muy variable. De igual manera, Peyton (1980) observó en el Perú, que de 25 cultivos de maíz, tres fueron completamente comidos por el oso y cinco solo parcialmente. En Ecuador comentaron que un oso podía acabar 1 ha de maíz en 15 días, otra persona estimó que de una producción de 4 ha que son 15,000 kg de maíz desgranado, el oso comió 1,000kg; otros agricultores perdieron toda su cosecha (Bejarano 1999).

Las agrupaciones temporales de los osos, en la época de cosecha para fines alimenticios, reportadas en Yanachaga Chemillén, Machu Picchu y Bahuaja Sonene, también fueron registradas anteriormente en maizales en Machu Picchu (hasta 10 osos) y tunales en Apurímac (hasta nueve osos; Peyton 1980). En Chucén (Cajamarca), se reportó un grupo de siete individuos (seis adultos y un osezno) alimentándose de yucas (Figueroa et al. 2013).

Hay que tomar en cuenta que, a diferencia de los cultivos de maíz para su venta a los grandes mercados, donde se aplica tecnología para obtener un mayor rendimiento de mazorcas por hectárea, en las áreas donde se realizaron las entrevistas los campesinos siembran el maíz en pequeñas parcelas que no cuentan con ningún soporte tecnológico ni vías de acceso para la salida de sus productos, y cuyos principales objetivos son el autoconsumo, intercambio por otros cultivos de zonas más altas (como por ejemplo papa) y alimento para sus animales de corral. Por ello, en estos casos, el ingreso de los osos a sus cultivos les origina una gran pérdida. Esto conlleva a que señalen al oso como un animal perjudicial, lo que motiva a su cacería cuando los ven cerca a sus propiedades e incluso si estos están en el bosque y no han ocasionado daños, o a usar cebos con plaguicida Paratión para envenenarlo, como ocurrió anteriormente en Machu Picchu (Peyton 1987).

\section{Depredación de ganado}

En base a los resultados de las entrevistas y los registros de otros autores, se tendría un total de siete especies de ganado como parte de la dieta del oso andino (Tabla 7). Si bien, existen investigaciones que confirman la depredación del ganado vacuno en Venezuela (Goldstein 1992), Colombia (Poveda 1999; Rodríguez et al. 2004), Ecuador (Castellanos 2002; Castellanos et al. 2011; Laguna 2013) y Bolivia (Nallar et al. 2008), en el Perú aún no se ha hecho un estudio al respecto. Sin embargo, 13 personas 
Tabla 4. Cultivos reportados como alimento del oso andino en las entrevistas.

\begin{tabular}{|c|c|c|c|c|c|c|c|c|c|c|c|c|c|c|c|}
\hline 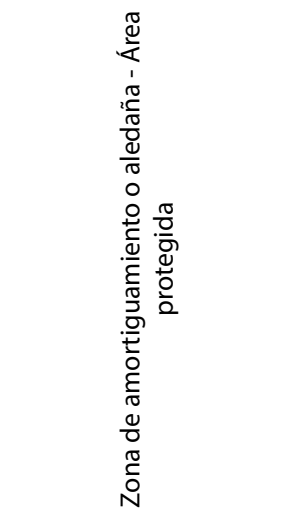 & 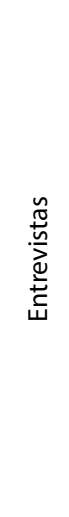 & 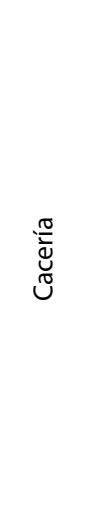 & 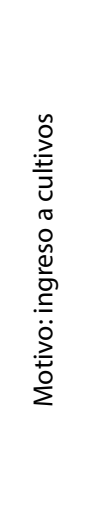 & 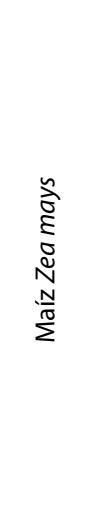 & 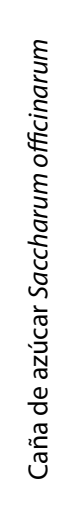 & 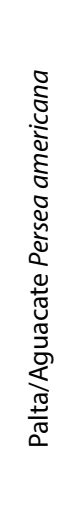 & 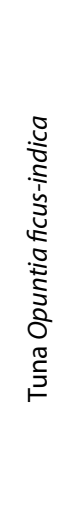 & 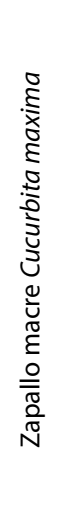 & 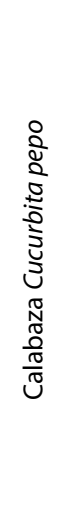 & 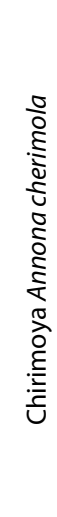 & 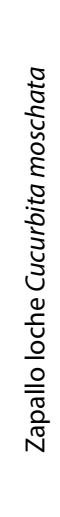 & 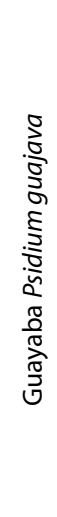 & 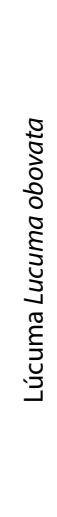 & 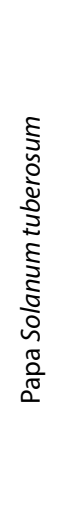 & 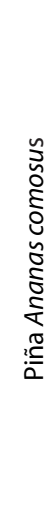 \\
\hline Huaylla Belén-Colcamar & 16 & 56.3 & 55.6 & 100.0 & & & & & & & & & & & \\
\hline Huamanpata & 6 & 83.3 & 60.0 & 100.0 & & & & & & & & & & & \\
\hline Huascarán & 17 & 88.2 & 6.7 & 100.0 & & & & & & & & & & & \\
\hline Choquequirao & 16 & 56.3 & 88.9 & 67.7 & & 12.4 & 19.9 & & & & & & & & \\
\hline Cutervo & 31 & 9.7 & 66.7 & 80.2 & 19.8 & & & & & & & & & & \\
\hline Machu Picchu & 26 & 76.9 & 70.0 & 83.9 & 4.1 & 9.2 & & & & & & & & & 2.8 \\
\hline Manu & 11 & 90.9 & 70.0 & 96.8 & & & & & & & & & & 3.2 & \\
\hline Megantoni & 15 & 46.7 & 14.3 & 100.0 & & & & & & & & & & & \\
\hline Tingo María & 19 & 21.1 & 100.0 & 100.0 & & & & & & & & & & & \\
\hline El Sira & 28 & 10.7 & 86.7 & 100.0 & & & & & & & & & & & \\
\hline Calipuy & 8 & 100.0 & & & & & & & & & & & & & \\
\hline Laquipampa & 25 & 52.0 & 7.7 & 84.6 & & & & & & 6.2 & 3.8 & 2.9 & 2.5 & & \\
\hline San Matías-San Carlos & 8 & 25.0 & 20.0 & 100.0 & & & & & & & & & & & \\
\hline Yanachaga Chemillén & 54 & 70.4 & 71.1 & 85.6 & & & & 7.8 & 6.6 & & & & & & \\
\hline Bahuaja Sonene & 7 & 85.7 & 50.0 & 100.0 & & & & & & & & & & & \\
\hline Alto Mayo & 23 & 100.0 & 8.7 & 100.0 & & & & & & & & & & & \\
\hline Total ( \%) & 310 & 60.8 & 48.5 & 93.3 & 1.6 & 1.4 & 1.3 & 0.5 & 0.4 & 0.4 & 0.3 & 0.2 & 0.2 & 0.2 & 0.2 \\
\hline
\end{tabular}

proporcionaron información muy detallada de sus observaciones, las cuales coincidieron con otras sobre el ataque del oso al ganado, hechas en diversas zonas del Perú (Peyton 1980; Figueroa et al. 2013) y en otros países (Goldstein et al. 2006; Castellanos et al. 2011), lo que confirmaría que esta depredación también se da en el Perú.

Lamentablemente, la percepción del oso como depredador de ganado está muy generalizada, a pesar que la mayoría de los entrevistados señalaron que no presenciaron directamente el ataque, sino que refieren los comentarios de terceros o que observaron el consumo de la carroña. En algunos casos, estos comentarios tienden a magnificarse, como se ha visto en otros estudios relacionados a la cacería del oso andino basados en entrevistas (Torres et al. 1995; Balarezo 1999), o podrían ser exageraciones de los campesinos locales para justificar la cacería de la especie (Peyton 1980; Goldstein 1991; Poveda 1999).

La pérdida del ganado podría deberse a muchos otros factores, como enfermedades, accidentes e incluso robo por abigeos, sin embargo, la fama de depredador de ganado vacuno se afianza al encontrar heces, rasguños o signos de alimentación de los osos alrededor del cadáver (Peyton 1980; Goldstein 1991, 1992; Poveda 1999; Paisley 2001). 



Figura 5. Reportes de ataque del oso andino al ganado vacuno. a) Otocani (zona de amortiguamiento de Manu), se observan rasguños dejados por el oso (Foto: ProBosque Manu/Sociedad Zoológica de Frankfurt Perú). b) Pacopampa (Apurímac; Foto: Jan Baiker/ Ecobona-Cosude). c) Otocani (Foto: ProBosque Manu/Sociedad Zoológica de Frankfurt Perú). d) Mishkiuno (zona de amortiguamiento de Megantoni), oso cazado por evento de depredación, se pueden observar los dientes muy desgastados (Foto: Judith Figueroa).

Estos registros no constituyen necesariamente una prueba de depredación, sino más bien de alimentación de huesos y carne (Paisley 2001). Asimismo, el reporte de los dientes desgastados en algunos osos cazados, tampoco comprobaría la depredación del ganado. Si bien las fracturas y roturas en el esmalte estarían relacionadas con la masticación de huesos (Soibelzon 2002), esto podría deberse a un evento carroñero. El desgaste y ruptura de los dientes también podrían estar relacionados con la edad y una dieta rica en carbohidratos (Stucchi y Figueroa 2013).

Por ello, se debe de considerar de manera prioritaria la diferenciación de los reportes de interacción oso-ganado, entre depredación o carroña, para conocer el alcance real del primero en el área evaluada. Peyton (1980) revisó 14 cueros de ganado vacuno cuyas muertes fueron atribuidas al oso andino, las marcas de los rasguños mostraron que nueve correspondían a pumas. En el Parque Nacional de Chingaza (Colombia) los pobladores reportaron 18 vacas supuestamente atacadas por osos en 1996 y 43 muertas por la misma causa en 1997; después del trabajo de verificación de Poveda (1999), los reportes disminuyeron drásticamente pasando a cinco y cuatro durante 1998 y 1999 , respectivamente.

Todas las áreas que fueron visitadas, donde los campesinos señalaron la depredación del ganado, estuvieron rodeadas de bosques, donde a partir de la tarde la visibilidad era muy baja debido a la espesa neblina. Todas estas condiciones serían favorables para el ataque sorpresa de un oso. Esta cercanía del bosque a los sitios de ataque también fue observada en Colepato (Parque Nacional 
Sangay, sur de Ecuador; Achig 2009). Sin embargo, en otras áreas de Ecuador y Venezuela, se registraron conflictos cerca de zonas ocupadas por humanos, en el segundo caso, en áreas abiertas ubicadas hasta $8 \mathrm{~km}$ del bosque (Goldstein et al. 2006).

A continuación, en base a las entrevistas y revisión bibliográfica, se comentan tres tipos de eventos que estarían relacionados con el consumo de carne de ganado por el oso andino:

Consumo como carroña del ganado muerto por causas externas a la depredación. Los hábitos carroñeros del oso andino han sido registrados en Ecuador. En la región Intag, un oso andino consumió los restos de un individuo de la misma especie (Castellanos 2006). En el Perú, en Machu Picchu, se alimentó de los restos de un perro y una llama (Figueroa 2013); en Lambayeque, de los restos de un venado de cola blanca Odocoileus virginianus (Van Russ et al. 2014); y en el presente estudio, de los restos de ganado vacuno (Huascarán y Calipuy) y un ave (Cutervo). En Machu Picchu los osos se desplazan por la puna (3,400-4,000 msnm) durante los meses de lluvias, entre noviembre y abril, en busca de Puya spp. y frutos maduros de Ericaceae, y en agosto, cuando no hay frutos en los bosques más bajos. En estos meses también se reporta la depredación al ganado (Peyton 1983). En la época de lluvias, debido a las condiciones climáticas y a la cosecha de papa y maíz, los campesinos suben solo un día cada dos semanas a la puna a darle sal a su ganado, por lo que señalan que el oso aprovecha esta circunstancia para atacar a los vacunos (Peyton 1980). Reportes similares se obtuvieron en el páramo del norte del Perú (Peyton 1987). Paisley (2001) encontró en Apolobamba (Bolivia) un mayor consumo de carne de vacuno por los osos durante la época de lluvias, esto fue relacionado con la posibilidad de que el ganado pueda resbalarse en los terrenos escarpados cuando buscan los brotes del pasto después de la quema al final de la estación seca.

Depredación del ganado por parte del puma y el consumo de los restos por parte del oso como carroña. En Quindio (cordillera central de los Andes, Colombia), durante dos años se obtuvieron 75 registros de ataques a ovejas, cabras, mulas, caballos, vacas y perros, perpetrados en su totalidad por pumas (Payán 2004). Este hecho llamó la atención, ya que se tenía la confirmación de eventos de depredación por parte del oso andino en la cordillera oriental (Rodríguez et al. 2004), y en la zona donde se dieron la mayoría de los ataques, entre los 2,800 y 3,000 msnm, habitaban el puma y el oso (Payán 2004). Algunas personas entrevistadas en el Perú (Cutervo, Huascarán y comunidad de Sandia), comentaron que el ganado es depredado por el puma y los restos consumidos como carroña por el oso. Una característica determinante del puma, es que esconde los restos de sus presas para protegerlas de otros depredadores, si la presa es de gran tamaño, la distribuye por diversos sitios (Nallar et al. 2008). Se conoce que el puma y el oso utilizan las mismas áreas (Goldstein 2002) e incluso los mismos senderos y árboles para la marcación de su territorio (Payán 2004; Figueroa 2005), por lo que debido al comportamiento alimenticio oportunista del oso, no sería raro este hecho.

En Glacier (EE.UU.), los osos pardo y negro americano ubican el $15 \%$ de las presas cazadas por el puma, apropiándose del $7 \%$ de estas; mientras que en Yellowstone, ubican el 33 \% y se apropian del $12 \%$ (Murphy et al. 1998). Entre Eslovenia y Croacia, los osos pardos encontraron y se apropiaron del $32 \%$ de las presas cazadas por el lince boreal Lynx lynx (Krofel et al. 2012). La disminución de las poblaciones de pumas podría también incidir en el comportamiento del oso andino como depredador, debido a la disminución de la carroña.

Ataque del oso al ganado para su consumo. No sorprende que algunos individuos de oso andino depreden ganado vacuno, ya que existen reportes del ataque y consumo a otros mamíferos grandes silvestres, como venado, guanaco, vicuña (Tschudi 1844 en Peyton 1980; Luscombe 1989) y tapir de montaña Tapirus pinchaque (Peyton 1980; Castellanos 2011; Rodríguez et al. 2014).

A pesar de obtener en las entrevistas diversas descripciones sobre el ataque del oso al ganado, estas se centran en dos técnicas. En la primera, el oso sorpresivamente persigue al rebaño hasta 
Tabla 5. Ganado depredado por el oso andino según las entrevistas.

\begin{tabular}{|c|c|c|c|c|c|c|c|c|}
\hline $\begin{array}{c}\text { Zona de } \\
\text { amortiguamiento } \\
\text { o aledaña - Área } \\
\text { protegida }\end{array}$ & 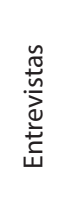 & 蹗 & 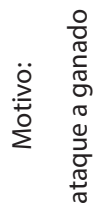 & 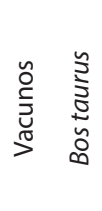 & 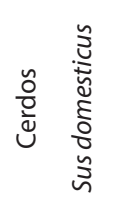 & 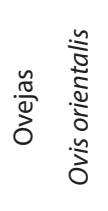 & 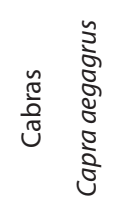 & 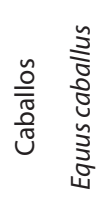 \\
\hline $\begin{array}{l}\text { Huaylla Belén- } \\
\text { Colcamar }\end{array}$ & 16 & 56.3 & 33.3 & 100 & & & & \\
\hline Huamanpata & 6 & 83.3 & 20 & 100 & & & & \\
\hline Huascarán & 17 & 88.2 & 93.3 & 69.4 & 6.8 & 7.3 & 7.3 & 9.2 \\
\hline Choquequirao & 16 & 56.3 & 11.1 & 100 & & & & \\
\hline Cutervo & 31 & 9.7 & 28.0 & 100 & & & & \\
\hline Machu Picchu & 26 & 76.9 & 20 & 100 & & & & \\
\hline Manu & 11 & 90.9 & 30 & 100 & & & & \\
\hline Megantoni & 15 & 46.7 & 85.7 & 100 & & & & \\
\hline Tingo María & 19 & 21.1 & & & & & & \\
\hline El Sira & 28 & 10.7 & 13.3 & 100 & & & & \\
\hline Calipuy & 8 & 100 & 100 & 100 & & & & \\
\hline Laquipampa & 25 & 52 & 92.3 & 85.1 & & & 14.9 & \\
\hline San Matias-San Carlos & 8 & 25 & & & & & & \\
\hline Yanachaga Chemillén & 54 & 70.4 & 23.7 & 88.2 & 3.3 & 8.5 & & \\
\hline Bahuaja Sonene & 7 & 85.7 & 33.3 & 100 & & & & \\
\hline Alto Mayo & 23 & 100 & 91.3 & 100 & & & & \\
\hline Total (\%) & 310 & 60.8 & 42.2 & 95.9 & 0.7 & 1.1 & 1.6 & 0.7 \\
\hline
\end{tabular}

guiar a uno al barranco para hacerlo caer. En este punto, se comentó incluso la participación de más de un oso. En la segunda, el vacuno solitario es sorprendido por el oso quien se trepa sobre su espalda, mordiéndole y rasguñándole el lomo, el área de los omóplatos, la nuca y la cola, hasta derribarlo. En el primer caso, se señaló que el oso come la presa en el mismo lugar, mientras que en el segundo, el oso arrastra los restos hacia un lugar más seguro dentro de la cobertura vegetal o hacia una plataforma construida por el oso para alimentarse. Solo este último coincide con los registros de Goldstein $(1992,2002)$ y Poveda (1999). Es muy probable que debido a la inaccesibilidad del área donde cayó el ganado desbarrancado este no pueda ser trasladado a un lugar más seguro. La relación de la construcción de la plataforma con eventos de depredación ha sido anteriormente registrada (Peyton 1980; Goldstein 1991, 1992, 2002; Castellanos 2002) y reportada en otras zonas (Figueroa et al. 2013).

Adicionalmente, Nallar et al. (2008) señalan que los osos matan a su presa con golpes y mordeduras en la cabeza y el cuello, ocasionando fracturas en los huesos del cráneo, cuello y hombros. La presa puede ser consumida aún estando viva, sin importar que esté en decúbito o parada (Castellanos 2002). Los osos además, pueden retirar casi por completo la piel de la presa y dejar sus huesos 
intactos (Goldstein 1992; Nallar et al. 2008). Los restos son movidos de un lugar a otro en varias oportunidades (Goldstein 1991, 2002).

A diferencia de los felinos, que se encuentran perfectamente conformados para la depredación de presas grandes por sus hábitos carnívoros: dientes modificados que cortan la carne, garras retráctiles para aferrarse y amplia apertura de la boca, el oso andino siendo filogenéticamente un carnívoro, ha experimentado cambios morfológicos a nivel del cráneo y mandíbula que le permiten alimentarse de una dieta omnívora, con preferencia en las plantas (Figueirido et al. 2009). Estas características conllevan a que el éxito de su ataque se base en su fuerza corporal, por lo que el tamaño del cuerpo del oso podría influir en la selección del tamaño de las presas. En consecuencia, los osos grandes pueden tener más probabilidades de matar al ganado adulto que los osos más pequeños. Esto se observó en la depredación de alces Alces alces y ganado por parte del oso pardo, la cual se limitada a osos machos grandes (Haglund 1968 en Zimmermann et al. 2003). Los osos machos más pequeños y las hembras podrían preferir el ataque a becerros, como se ha visto anteriormente en Ecuador y Colombia (Castellanos y Laguna 2012) y como se ha reportado en el presente estudio.

Esto podría explicar los reportes de depredación del ganado, principalmente por los osos andinos machos adultos en este estudio y en otras áreas (Castellanos et al. 2011). Los machos pueden llegar a medir hasta $2 \mathrm{~m}$ y pesar $200 \mathrm{~kg}$, mientras que las hembras llegan a los 2/3 de estas dimensiones (Peyton 1999; Castellanos 2010). Esto también podría explicar la ausencia de depredación de vacunos de la raza Brahman en Cosanga (Ecuador, Castellanos et al. 2011), debido a su gran talla con cabeza ancha y joroba en su lomo, llegando a pesar un macho adulto entre 800 a 1,100 kg y una hembra entre 450 y 600 kg; además de su comportamiento gregario por lo que pastan en grupos como medio de defensa, y al instinto maternal muy fuerte de las hembras, que protegen a las crías contra los enemigos naturales (Asocebú 2014).

En el caso que el ganado sea muy grande para el oso atacante, este podría preferir desbarrancarlo que enfrentarse directamente con él, para no salir lastimado. Esto se relacionaría con los comentarios de varios campesinos "el oso observa al vacuno sobre el cual va a saltar, para medirle la gordura y fuerza". Si bien la mayoría de los eventos de depredación de los osos se dan por individuos solitarios, en algunos reportes se describieron ataques grupales de osos, que incluyeron una pareja reproductora (Machu Picchu), y un grupo de tres osos (Alto Mayo) y otro de seis (Calipuy). Estos últimos podrían ser subadultos o hembras con crías de hasta dos años de edad trabajando de forma coordinada para la obtención de una presa. Estos ataques grupales de osos de diferentes edades también se registraron en otros úrsidos como el oso pardo en Yellowstone (parejas reproductoras, subadultos, hembras con oseznos y con crías mayores de un año; Gunther y Renkin 1990).

El mayor registro de depredación a ganado vacuno adulto (58.8 \%) sobre terneros (41.2 \%), podría responder a la teoría del forrajeo óptimo (Stephens y Krebs 1986), donde los osos deben comer las presas, o partes de la presa que proporcionan la mayor obtención de energía, para la supervivencia y el éxito reproductivo. En Noruega, se observó este comportamiento en el oso pardo hacia las ovejas, en áreas con alto número de presas; consumió principalmente la grasa de la caja torácica y las ubres (Knarrum et al. 2006). Para el caso del oso andino, los entrevistados reportaron el consumo de estas partes en algunas áreas y en otras las vísceras, al igual que en Cosanga (Ecuador, Castellanos 2002) y Apolobamba (Bolivia, Nallar et al. 2008). Por otro lado, también podría deberse al comportamiento agresivo de algunos vacunos en enfrentar a los depredadores y no huir, o a la tendencia de la vaca a proteger a su ternero contra las amenazas.

Los registros de ataques de hembras al ganado, podrían estar relacionados a las necesidades y exigencias de su organismo en obtener mayor calidad y cantidad de proteínas en la época de celo 
Tabla 6. Cultivos reportados como alimento del oso andino en su área de distribución.

\begin{tabular}{|c|c|c|c|c|c|c|c|c|}
\hline & Nombre común & Especie & Familia & Venezuela & Colombia & Ecuador & Perú & Bolivia \\
\hline 1 & Chirimoya & Annona cherimola & Annonaceae & & & & $\mathrm{Ra}^{\mathrm{f}, g}$ & \\
\hline 2 & Arracacha & Arracacia xanthorrhiza & Apiaceae & & & & $E n^{e}$ & \\
\hline 3 & Piña & Ananas comosus & Bromeliaceae & & & $\mathrm{Ra}^{\mathrm{b}}$ & $\mathrm{En}^{\mathrm{g}}$ & \\
\hline 4 & Tuna & Opuntia ficus-indica & Cactaceae & & & & $\mathrm{Ra}^{\mathrm{a}, \mathrm{d}, \mathrm{g}}$ & \\
\hline 5 & Calabaza & Cucurbita pepo & Cucurbitaceae & & & & $\mathrm{En}^{\mathrm{g}}$ & \\
\hline 6 & Zapallo macre & Cucurbita maxima & Cucurbitaceae & & & & $\mathrm{Ra}^{\mathrm{g}}$ & \\
\hline 7 & Zapallo loche & Cucurbita moschata & Cucurbitaceae & & & & $\mathrm{Ra}^{\mathrm{g}}$ & \\
\hline 8 & Yuca/Mandioca & Manihot esculenta & Euphorbiaceae & & & & $\mathrm{En}^{f}$ & \\
\hline 9 & Palta/Aguacate & Persea americana & Lauraceae & & & & $\mathrm{Rf}^{\mathrm{d}, \mathrm{g}}$ & \\
\hline 10 & Plátano/Banana & Musa paradisiaca & Musaceae & $\mathrm{En}^{\mathrm{a}}$ & & $\mathrm{Ra}^{\mathrm{a}}$ & $\mathrm{En}^{f}$ & $E n^{e}$ \\
\hline 11 & Cambur & Musa sapientum & Musaceae & $\mathrm{En}^{\mathrm{c}}$ & & & & \\
\hline 12 & Guayaba & Psidium guajava & Myrtaceae & & & $\mathrm{Ra}^{\mathrm{a}}$ & $E n^{g}$ & $E n^{e}$ \\
\hline 13 & Caña de azúcar & Saccharum officinarum & Poaceae & $\mathrm{En}^{\mathrm{a}}$ & $\mathrm{En}^{\mathrm{a}}$ & $\mathrm{Ra}^{\mathrm{a}}$ & $\mathrm{Ra}^{\mathrm{a}, \mathrm{f}, \mathrm{g}}$ & $\mathrm{En}^{\mathrm{c}}$ \\
\hline 14 & Maíz & Zea mays & Poaceae & $E^{a, b}$ & $\mathrm{En}^{\mathrm{a}}$ & $R a^{b, c}$ & $\mathrm{Ra}^{\mathrm{a}, \mathrm{b}, \mathrm{c}, \mathrm{f}, \mathrm{d}}$ & $\mathrm{En}^{\mathrm{b}, \mathrm{d}}$ \\
\hline 15 & Naranja & Citrus sp. & Rutaceae & & & & & $\mathrm{En}^{\mathrm{a}}$ \\
\hline 16 & Lúcuma & Lucuma obovata & Sapotaceae & & & & $\mathrm{Ra}^{\mathrm{a}, \mathrm{f}, \mathrm{g}}$ & \\
\hline 17 & Naranjilla & Solanum quitoense & Solanaceae & & & $\mathrm{Ra}^{\mathrm{b}}$ & & \\
\hline 18 & Papa & Solanum tuberosum & Solanaceae & $\mathrm{En}^{\mathrm{a}}$ & $E n^{a}$ & & $\mathrm{En}^{\mathrm{g}}$ & \\
\hline
\end{tabular}

En: Entrevista. Ra: Registro alimenticio. Rf: Registro fotográfico. Venezuela: a) Herrera et al. 1994 (Parque Nacional Sierra Nevada); b) Torres 2006; c) Lameda y Monsalve com pers. en Castellanos 2010. Colombia: a) Perico y García 2001 en Ojeda y Pesca 2006 (Mamapacha, Boyacá). Ecuador: a) Castellanos et al. 2005 (Reserva Biológica Maquipucuna y Parque Nacional Sangay); b) Castellanos 2010 (Intag y Cosanga); c) Bejarano 1999 (entre Baeza y Cosanga). Perú: a) Peyton 1980; b) Butchart et al. 1995 (Cordillera de Colán, Amazonas); c) Tschudi 2003 [18381842]; d) Baiker 2011 (Bosque de Chinchay y Pilcomarca, Apurímac); e) Figueroa y Stucchi 2013 (Corredor Vilcabamba-Amboró);f) Figueroa et al. 2013 (áreas aledañas al río Marañón, Cajamarca-Amazonas); g) Presente estudio. Bolivia: a) Yáñez y Eulert 1996 (Serranía de Los Milagros, Chuquisaca); b) Rumiz et al. 1999 (Parque Nacional Amboró y Carrasco); c) Paisley 2001 (Pusupunko); d) Albarracín 2010 (Chuñavi y Lambate); e) Ríos-Uzeda y Zenteno com pers. en Castellanos 2010.

y del cuidado de las crías en áreas con recursos alimenticios limitados. Al respecto, en un estudio en cautiverio, se encontró una variación significativa en la dieta y comportamiento de la hembra en los meses de los picos reproductivos, donde aumentó radicalmente el consumo de proteínas, e incluso se registraron arrebatamientos de carne al macho. Este cambio de conducta llamó la atención, ya que normalmente el macho siempre se mostró dominante en la elección e ingestión de la carne sobre la hembra (Figueroa y Stucchi 2003).

En algunos casos, los reportes de ataques de un mismo individuo de oso andino durante un lapso de tiempo en un área (presente estudio; Goldstein 1992), coinciden con los patrones reportados para especies de otros carnívoros (Treves et al. 2004). En el caso del oso pardo, el riesgo de un segundo ataque a ovejas a la siguiente semana, en una misma área, fue de $47 \%$ y en la quinta semana de $63 \%$. Esto podría deberse a que el depredador regresa al mismo sitio para alimentarse de la carroña o para buscar nuevas presas. Por ello se recomendó implementar medidas disuasivas en las fincas dentro de las cinco semanas del primer evento (Karlsson y Johansson 2010). En otros casos, como en el norte de Ecuador, diferentes osos atacaron al ganado vacuno en un mismo sector y periodo (Castellanos y Laguna 2012; Laguna 2013). Estos ataques estarían relacionados con el éxito del oso en ocultar su presa para evitar interactuar con otros carnívoros como pumas, zorros, perros asilvestrados, u otros osos, en este caso tendría entre 8 y 10 días para consumirla; en el caso de que sea arrebatada por algún competidor, el oso emprenderá una nueva cacería (Laguna 2013). 
Tabla 7. Ganado reportado como parte de la dieta del oso andino.

\begin{tabular}{|c|c|c|c|c|c|c|c|}
\hline & Nombre común & Especie & VE & CO & EC & PE & BO \\
\hline 1 & Caballo & Equus caballus & & & $\mathrm{Ra}^{\mathrm{d}, \mathrm{g}}$ & $E^{d}$ & $\mathrm{Ra}^{\mathrm{d}, \mathrm{e}}$ \\
\hline 2 & Mula & Equus caballus $x E$. asinus & & & & & $\mathrm{En}^{\mathrm{d}}$ \\
\hline 3 & Cerdo & Sus domesticus & & & & $E n^{d}$ & \\
\hline 4 & Vacuno & Bos taurus & $\mathrm{Ra}^{\mathrm{a}, \mathrm{b}}$ & $\mathrm{Ra}^{\mathrm{a}, \mathrm{b}}$ & $\mathrm{Ra}^{\mathrm{a}, b, c, d, e, f, g}$ & $\mathrm{Ra}^{\mathrm{a}, \mathrm{d}}$ & $\mathrm{Ra}^{\mathrm{b}, \mathrm{d}, \mathrm{e}}$ \\
\hline 5 & Cabra & Capra aegagrus & $\mathrm{En}^{\mathrm{b}}$ & & & $\mathrm{Ra}^{\mathrm{a}, \mathrm{d}}$ & \\
\hline 6 & Oveja & Ovis orientalis & $\mathrm{En}^{\mathrm{a}, \mathrm{b}}$ & & $\mathrm{Ra}^{\mathrm{d}}$ & $\mathrm{En}^{\mathrm{b}, \mathrm{d}}$ & \\
\hline 7 & Llama & Lama glama & & & & $\mathrm{Rf}^{\mathrm{c}}$ & $\mathrm{En}^{\mathrm{c}}$ \\
\hline
\end{tabular}

En: Entrevista. Ra: Registro alimenticio. Rf: Registro fotográfico. Venezuela: a) Goldstein 1989; b) Herrera et al. 1994 (Parque Nacional Sierra Nevada). Colombia: a) Poveda 1999 (Parque Nacional Natural Chingaza); b) Rodríguez et al. 2004 (Mamapacha, Bijagual, San Cayetano, Ubala, páramo de Guasca y Chingaza). Ecuador: a) Suárez 1988 (Volcán Antisana, 3550-4500 msnm); b) Bejarano 1999 (Entre Baeza y Cosanga); c) Castellanos et al. 2005 (Reserva Biológica Maquipucuna, 1200-2700 msnm y Parque Nacional Sangay); d) Flores et al. 2005 (Oyacachi); e) Castellanos 2010 (regiones de Intag y Cosanga); f) Castellanos et al. 2011 (Cosanga); g) Laguna 2013 (norte de Ecuador). Perú: a) Peyton, 1980: varias áreas del Perú; b) Figueroa 2008 (Carpish, Huánuco); c) Figueroa 2013 (Machu Picchu); d) Presente estudio. Bolivia: a) Yáñez y Eulert 1996 (Serranía de Los Milagros, Chuquisaca); b) Rumiz et al. 1997; c) Rumiz et al. 1999 (Parque Nacional Amboró y Carrasco, 550-3700 msnm); d) Paisley 2001 (Pusupunko, 2700-4300 msnm); e) Rivadeneira 2001 (región Apolobamba, 2800-4000 msnm).

\section{Relación entre la disponibilidad de los frutos silvestres con el ataque al ganado e ingresos a los cultivos}

Los daños ocasionados por el oso andino fueron diferentes en cada localidad. Esta variación también se presentó en muchas otras especies de carnívoros grandes (Treves 2009). En algunas zonas el oso andino ingresó a comer diversos tipos de cultivos, mientras que en otras, se limitó solo al maíz, a pesar de que también estaban presentes los cultivos de los cuales se alimentan en otras áreas.

Por otro lado, existen áreas donde no se reportó la depredación del ganado, desde la década de 1990, como en Laquipampa y Cutervo. En las comunidades aledañas al río Marañón, entre Cajamarca y Amazonas, que fueron entrevistadas el 2012, los eventos más recientes fueron en el 2003. En algunas localidades de La Libertad esta depredación se dio entre 1982 y 2002 (Figueroa et al. 2013). En Alto Mayo, la depredación se registró solo en las comunidades del lado de Amazonas y no hacia el lado de San Martín (presente estudio; Vela 2008). En San Miguel (Machu Picchu) no se reportó ningún evento de depredación a pesar de ser una zona ganadera (Figueroa y Stucchi 2013). En Colombia, el mayor número de eventos de ataques al ganado y consumo de cultivos se dieron en la cordillera occidental (29), seguida de la oriental (27) y la central (10; Jorgenson y Sandoval 2005).

De la misma manera, las frecuencias también variaron. En algunas áreas de Venezuela se reportaron eventos predatorios en un intervalo de 10 años (Goldstein 1992). En el norte de Ecuador, entre 2009 y 2013, se registraron periodos de tres meses (abril - junio) en los que un oso macho dejó de atacar al ganado (Laguna 2013).

Algunos estudios en otros úrsidos atribuyeron las interacciones ganado-oso y cultivo-oso a una menor disponibilidad de alimento silvestre (Witmer y Whittaker 2001). En el caso del oso solar, los daños causados en los cultivos fueron mayores después del Evento El Niño 19971998 , en donde hubo una gran sequía y varios incendios forestales, lo que redujo su hábitat y disponibilidad de los frutos (Fredriksson 2005). Por otro lado, se encontró que los daños del oso negro americano a los cultivos cesaron cuando los frutos silvestres que forman parte de su dieta 
se encontraron más disponibles (Ziegltrum y Nolte 1996). En Yellowstone, Harting (1985 en Gunther y Renkin 1990) observó que los osos pardos depredaron menos mamíferos grandes en las zonas con mayor abundancia de alimentos vegetales.

Figueroa (2013) realizó una revisión de las crónicas de los españoles escritas a partir del siglo XVI sobre los registros del oso andino, no encontrando ninguna relacionada al ingreso a los cultivos de maíz en la época incaica. Incluso, en las descripciones detalladas de Guamán Poma de Ayala (2004 [1615-1616]) sobre la siembra, cuidado y cosecha del maíz en esa época, solo se nombraron como animales perjudiciales de este cultivo a las llamas, zorros, venados, zorrinos, perros, tordos, perdices, loros y papagayos. Al parecer los ingresos de los osos a los maizales fueron muy poco comunes, a pesar de haber sido ampliamente extendido su cultivo en la época incaica (Inocente et al. 2006). Con la llegada de los españoles, se produjeron rápidos cambios debido a la extracción de los recursos (Rostworowski 2005), lo que pudo ocasionar una disminución de las fuentes de alimento para el oso en algunas zonas, forzándolo a ingresar a los cultivos de maíz. Posteriormente, el crecimiento poblacional y la expansión de la frontera agrícola, podría haber influido en la frecuencia de estos ingresos en relación a la disminución de su alimento natural o al fácil acceso a los nuevos recursos alimenticios cultivados.

Con respecto al ataque de los osos andinos al ganado, Humboldt (2004 [1801-1802]) señaló que este solo se daba cuando no disponían de las plantas de las que se alimentaban naturalmente. Poveda ( $\mathrm{s} / \mathrm{f})$ encontró que en las zonas donde el bosque estaba mejor conservado y más continuamente distribuido, a pesar de haber ganado, los ataques no fueron un comportamiento habitual por parte del oso. Sin embargo, en el Perú sí se reportaron ataques en bosques en muy buen estado con abundantes recursos alimenticios, lo que podría estar relacionado a una mayor facilidad y disponibilidad de presas.

\section{Medidas que buscan reducir la cacería del oso andino}

Compensación. La compensación monetaria para la protección de los osos como retribución por los daños causados a los cultivos y ganado, a cambio de que no sea cazado, ha sido un éxito en algunos programas, como el del oso pardo en Europa (Klenzendorf 1997). Esta fue tomada en cuenta para el oso andino dentro del "Plan de acción para la conservación de los osos" (Peyton 1999). Sin embargo, en algunos casos podría tornarse conflictivo debido al uso de disposición a pagar/aceptar, que se refiere a la forma cómo se debe abordar una situación que necesite de una política, porque no está resuelto si se debe aplicar desde una perspectiva de compensación o desde una de adquisición (Pearce y Turner 1990 en Bejarano 1999).

En Ecuador, la Corporación de Conservación y Desarrollo (CCD 1994) elaboró un estudio sobre la factibilidad de realizar una compensación financiera por los daños que hicieran los osos en Cuyuja, Sardinas y Bermejo (Napo). El análisis de las encuestas y las cifras de comercialización concluyeron que el maíz no constituía la fuente principal de subsistencia para los agricultores y que los daños producidos sobre los cultivos por los osos eran mínimos. Se encontró que la caza y el comercio del oso andino eran más rentables, en términos económicos, que la producción y comercialización del maíz. Por ello, se determinó que la razón de sembrar maíz era para cazar al oso (CCD 1994). En Colombia tampoco se recomendó la compensación económica por los daños al ganado debido a la dificultad de diferenciar su depredación o carroñeo (Jorgenson y Sandoval 2005). Sin embargo, actualmente para el norte de Ecuador, debido a la fuerte tendencia creciente de los ataques (Laguna 2013), se evalúa la posibilidad de crear fondos de compensación por la pérdida de ganado, lo que podría influir de forma positiva en la tolerancia de estos eventos (Castellanos et al. 2011). En Oyacachi, entre los años 2001 y 2004, se registraron 41 eventos de ataques que dieron muerte a 61 vacunos, lo que representó una pérdida de USD\$31,516 en carne y USD\$ 42,534 en leche, para las familias 
afectadas (Flores et al. 2005). En las provincias de Carchi e Inambura, entre noviembre de 2009 y mayo de 2012, se comprobaron 163 ataques a ganado (141 muertos y 22 heridos; Castellanos y Laguna 2012), y hasta marzo de 2013, se registraron 98 eventos adicionales, sumando un total de 261 ataques (Laguna 2013). En el sur de Ecuador (Colapato), entre 1998 y 2008, se reportaron 40 vacunos muertos y uno herido (Achig 2009).

Otro tipo de compensación monetaria, podría estar relacionada con un ecoturismo basado en la observación del oso andino alimentándose en los maizales en la época de producción del choclo. El campesino sería compensado por su pérdida del maizal y por el tiempo que dedicaría al cuidado de los cultivos cuando no hay turistas (Peyton 1994).

Mitigación. El control letal lejos de ser un método efectivo, preventivo o correctivo, para la reducción de las interacciones humano-oso andino, podría conllevar a impactos negativos en sus poblaciones. Se analizaron varios estudios sobre la efectividad de la cacería de grandes carnívoros en la disminución de los daños ocasionados por estos a la ganadería; debido a la gran complejidad de los casos de depredación, se determinó que los datos científicos aún son escasos para tomar en consideración esta política de manera general (Treves 2009).

En Japón, no se encontró asociación entre los costos de los daños ocasionados por el oso negro asiático y su cacería, a pesar de que se cazaron más de 1,000 individuos cada año (Huygens et al. 2004). Respecto al oso negro americano, si bien en el Parque Nacional Fundy (Canadá) hubo una reducción de los conflictos después de su cacería, en otras tres áreas, dos en EE.UU. y una en Canadá, no se obtuvo el mismo efecto (Treves 2009; Treves et al. 2010).

Estudios en el oso pardo en cuatro áreas de caza entre la frontera de Canadá y EE.UU., sugirieron que la población era más susceptible a su disminución e incluso a una extinción rápida. Esto se debía a que la cacería de los machos adultos ocasionaba un aumento de la inmigración de nuevos machos, que reducía la supervivencia de los oseznos debido al infanticidio. Además, las hembras al evitar a los machos inmigrantes también se alejaban de los hábitats ricos en alimentos, lo que daba lugar a una disminución del tamaño de la camada (Wielgus y Bunnell 2000; Wielgus et al. 2001).

Por otro lado, lejos de lo esperado, la caza deportiva de pumas en Washington (EE.UU.) aumentó drásticamente los casos de depredación del ganado de un 36 a $240 \%$. Se sugirió que estos hechos estuvieron relacionados con el aumento de la inmigración de los machos jóvenes, que eran más propensos a atacar al ganado, en sustitución de los machos residentes cazados (Peebles et al. 2013).

Existen varios estudios donde se demostró que los carnívoros aprenden rápidamente a evitar las fincas después de aplicar diversos métodos de disuasión, debido a que los estímulos aversos se activan en respuesta a un comportamiento indeseable (Treves 2009). Para el caso del oso andino, existen documentos muy detallados donde se dan una serie de recomendaciones para la reducción de las interacciones con el maíz (Morales 2003) y el ganado (Nallar et al. 2008; Castellanos et al. 2011) cuya experiencia e información deben de ser tomados en cuenta.

Como punto inicial para plantear alternativas de mitigación que se ajusten a la realidad de cada área, será importante trabajar participativamente durante todo el proceso con los campesinos, revalorar sus conocimientos, comprender su temor hacia la pérdida económica, la percepción que tienen del animal que causó el daño, conocer el manejo de los agricultores y ganaderos, explicarles los objetivos y alcances del proyecto, y colectar información científica, para juntos buscar una solución a los conflictos y hasta prevenirlos.

Se recomienda la organización de las comunidades para las vigilancias de los campos de cultivos (Morales 2003) y del ganado (Castellanos et al. 2011). Este cuidado es básico e incluso es el control de mitigación más económico (Morales 2003). En la época incaica y colonial, se realizó 
una participación muy activa de hombres y mujeres en el cuidado de los maizales, principalmente en octubre (semillas recién sembradas), febrero (producción del choclo) y marzo-abril (maduración). Los hombres cuidaban durante el día y las mujeres, a partir de febrero, durante la noche, haciendo ruidos con un tambor (Guamán Poma de Ayala 2004 [1615-1616]). Con los años estas prácticas se fueron perdiendo en algunas zonas, y los cultivos ya no son atendidos continuamente, entre varios factores, porque se encuentran en áreas lejanas (Peyton 1999).

Para el caso de los ingresos del oso andino a los maizales hay que tomar en cuenta algunas observaciones realizadas anteriormente por Peyton (1980) en el Perú. Este autor encontró que los osos normalmente se alimentaron del maíz dos semanas antes de que esté maduro. Si un oso no fue asustado de un cultivo era probable que regresase al día siguiente, de lo contrario, se alimentaban de frutas y bromelias en el bosque o iban a otro maizal. Si el oso era asustado, podía regresar entre dos a 10 días después al mismo lugar. Además, observó que algunos mecanismos no detuvieron el ingreso del oso a un cultivo de maíz en Machu Picchu. El oso ingresó a pesar de la presencia de banderas azules de plástico en el perímetro, y regresó al día siguiente de la quema de caucho en el área donde ingresó el primer día.

Una experiencia exitosa de mitigación de daños a cultivos de maíz por el oso, gracias a la organización comunal, se realizó en Bolivia, en las comunidades de Pajan, K'apna y Wayrapata. En el 2001, 39 familias perdieron aproximadamente 6,701.8 kg de maíz (USD\$1,718.40), siendo consumidas por los osos, loros Aratinga mitrata, zorrinos Conepatus chinga y roedores Phylotis oscilae. Después de realizar un cuidado más intenso de los maizales (día y noche), con ahuyentamiento sonoro con petardos, ahuyentamiento visual con banderines plásticos de colores, y trampeo de ratones, el daño fue notablemente menor. Para el 2002, 39 familias perdieron 1,508.2 kg (USD\$385.97), y el 2003, 35 familias perdieron 2,982.3 kg (USD\$763.8), siendo los más perjudiciales los roedores, las aves y en menor grado los osos. También se recomendó el uso de espantapájaros y la limpieza del barbecho en los alrededores de cada parcela (Morales 2003).

Castellanos et al. (2011) sugieren varias medidas específicas para contrarrestar las interacciones del oso con el ganado vacuno en la región de Cosanga (Ecuador), en base a una investigación detallada en la zona. Una de ellas, es considerar la posibilidad de uniformizar las razas de ganado que se crían, por la raza Brahman, debido a que no se registraron eventos de su depredación. Otra, es no conceder permisos para la explotación de las lauráceas, ya que se encontró una estrecha relación del oso con esta familia: alimentación, refugio y marcaje. Esta recomendación también podría extenderse a otras áreas en el Perú, como Machu Picchu, en donde Peyton (1987) también observó esta relación.

Asimismo, existen diversas experiencias y conocimientos que se han producido en otros países relacionados con otros úrsidos que podrían tomarse en cuenta. En Noruega, se encontró un mayor riesgo de depredación por el oso pardo a las ovejas que llevaban campanas, ya que de alguna manera los primeros asociaron su sonido con el alimento (Knarrum et al. 2006). En Bolivia, este uso fue recomendado por Nallar et al. (2008) para alertar a los dueños de los movimientos bruscos del ganado debido a algún depredador, en especial por la noche. Será importante tomar en cuenta las condiciones del lugar para analizar el uso de este método. En Borneo, para el oso solar, se aplicó tiabendazol a los frutos de los huertos que fueron visitados por los osos, con el fin de repelerlos al relacionar a los frutos con un mal sabor (Fredriksson 2005). En la India, para el oso negro asiático, se recomendó el uso de cercas de alambre con tiras de plástico (Chauhan 1999). En Venezuela, en un área aledaña al Parque Nacional Sierra de la Culata, para mantener seguro al ganado vacuno, se usó cerco eléctrico, obteniendo buenos resultados iniciales (Vineyard y Torres 2004); sin embargo en el Perú, su aplicación es poco probable por los limitados recursos económicos de los campesinos.

Chauhan (1999) aconsejó el uso de algunos repelentes clásicos para mamíferos, como la capsaicina (componente activo de los pimientos picantes), el isotiocinato de alilo (esencia de mostaza), benzoato 
de denatonio y otros amargantes. La capsaicina es utilizada eficazmente en EE.UU. para repeler a los osos negros y pardos en los encuentros con humanos (Hunt 1984 en Clarkson y Stirling 1994). Este conocimiento se podría ajustar a la realidad de las comunidades del Perú, sembrando alrededor de los cultivos, bajo la supervisión de botánicos, barreras de vegetación que posean olores repelentes para los osos e ir probando su efectividad en la reducción de los daños. En la comunidad de El Chairo (Bolivia), para repeler de sus cultivos mixtos de yuca, walusa, maíz y plátano a aves y mamíferos, se utiliza como métodos tradicionales de control, el orín y la siembra de plantas de ají y flores junto con los cultivos (Morales 2003).

En algunos países del centro y sur de Europa, y en Asia, se han utilizado por milenios a los perros pastores como un medio eficaz de protección del ganado de los depredadores (Rigg 2001). En EE.UU., este método de vigilancia se introdujo en la década de 1970, teniendo muy buenos resultados en la actualidad, reduciendo la depredación de ovejas en un $11 \%$ a $100 \%$, dependiendo del lugar (Gehring et al. 2010 en Urbigkit y Urbigkit 2010). Además, los perros mantienen distantes a algunos animales que ocasionan daños a los cultivos, como los venados (Otstavela et al. 2009). El uso de perros entrenados de las razas Karelians y Akitas, ha sido anteriormente sugerido para disminuir la depredación del oso andino (Castellanos et al. 2011). Se podría explorar en el Perú, la idoneidad de los perros entrenados para la prevención de los daños de grandes carnívoros.

Hay que tomar en consideración que el oso andino puede tornarse agresivo para el hombre cuando es perturbado en un evento de depredación, al querer defender su presa. Además de los dos casos comentados en este estudio, Castellanos (2002) también reportó este comportamiento en dos ocasiones en Cosanga (Ecuador). Por ello, es sumamente importante recomendar a los pobladores, no interrumpir un evento de depredación o carroñeo.

La cacería del oso andino está registrada en todas las regiones políticas donde se distribuye el oso andino en el Perú, esta es motivada principalmente por las interacciones cultivo-oso y ganado-oso (Peyton 1980; Figueroa 2008; Figueroa et al. 2013). Debido al aumento de la frontera agrícola y ganadera, el territorio del oso disminuye rápidamente y con esto, la posibilidad de las interacciones se eleva (Peyton 1999). Las costumbres tradicionales de las comunidades en la agricultura y ganadería se encuentran muy arraigadas, por lo que el planteamiento de cambios drásticos no será fácilmente acogido por ellos. La alternativa más realista es la aplicación de técnicas de mitigación de daños acorde con las condiciones de cada lugar, en la que el campesino pueda darse cuenta que la prevención le originará una mayor ganancia económica y una mejor calidad de vida para su familia. Este es uno de los principales temas de investigación, donde se deberían dirigir los recursos económicos estatales y privados, para la conservación de la especie.

\section{Agradecimientos}

Un agradecimiento especial a J. Zaldivar, A. Vásquez, I. Pérez, M. Díaz, V. Cruzado y E. Vásquez (Cutervo); H. Cristóbal, H. Chamorro, L. Quicha, A. Sebastián, M. Soto, T. Ciriaco, A. Utani, E. Blásido, E. de la Cadena, C. Rojas, C. Mateo, J. Panti, D. Vásquez (Yanachaga Chemillén); L. Huanca, P. Suárez, R. Cañahuiri, I. Mamani, L. Huamanchoque y U. Churana (Manu); D. Huamán, L. Mamani, F. Suta, F. Puma, G. Martínez, J. Mendoza, R. Bello, F. Senperi, A. Nochomi, L. Camparo, R. Rivas, G. Manugari, R. Gutiérrez, N. García, J. Huallpa y H. Caparó (Megantoni); M. Manayay, N. Durand, M. Soplopuco, E. Flores, P. Mesones, J. Cabrera (Laquipampa); J. López, D. Sulca, R. Quispe, P. Cárdenas, C. Quispe, W. Danz, M. Baca, L. Contreras, F. Chávez, Y. Romero, C. González, J. Huayllapuma, W. Yucra y M. Pastor (Machu Picchu); J. Rebaza, V. Ruiz, O. García, P. Godoy y F. Mozo (Calipuy); S. Pinedo, J. Palomino, F. Aranda y A. Quispe (Tingo María); R. Valencia, C. Pineda, G. Suárez, S. Valverde, L. Champa, R. Narvaes, C. Alegre, N. Santillán, M. Zapata, C. Rodríguez, J. Gómez y W. Tamayo (Huascarán); A. Saldaña (Huamanpata). A T. Tapia por su apoyo en las entrevistas en Sandia. A M. 
Stucchi por la revisión del manuscrito y sus valiosos comentarios. A R. Rojas Vera-Pinto, J. Baiker, O. Mujica, R. Gutiérrez, A. Valdeiglesias y M. Cabrera por el préstamo de sus fotografías. A S. Kastl de la Cooperación Técnica Alemana y R. Rojas del Centro para la Conservación y Desarrollo Sostenible del Jardín Botánico de Missouri, por el financiamiento.

\section{Resumen}

Introducción: El oso andino Tremarctos ornatus fue cazado durante la época incaica por ser considerado como un animal dañino, depredador de vicuñas y guanacos. Posteriormente, con la llegada de los españoles, el ganado vacuno traido por ellos, proporcionó una nueva y abundante fuente de alimento para los osos. Esto conllevó a fortalecer la percepción de los pobladores hacia el oso como depredador, sin tener en cuenta que también podría tratarse de eventos carroñeros. Asimismo, se extendieron los cultivos de maíz en donde el oso también aprovechó de estos. En la actualidad se siguen reportando estas interacciones. El presente trabajo busca, en base a entrevistas a los campesinos, obtener información sobre cómo, cuándo y dónde se producen estas interacciones, así como el de conocer su percepción sobre la especie. Asimismo, se discute el conocimiento de estas interacciones en otros úrsidos y las experiencias de mitigación en otros países.

Metodología: Entre los años 2002 y 2008 se visitaron 50 comunidades de 12 regiones del Perú, ubicadas en áreas aledañas o en las zonas de amortiguamiento de 16 áreas protegidas con registros de presencia del oso andino. Se realizaron 310 entrevistas personales, principalmente a cazadores, agricultores y ganaderos. Se aplicó una entrevista semi-estructurada, en la que se preguntó básicamente: fecha, hora, lugar y tipo de interacción (ganado o cultivo), tipo de registro (observación, rastros), especie de cultivo consumido o animal depredado, descripción del evento (ingresaron osos solitarios o en grupos, edades de los osos, etc.) y motivo de la cacería.

Resultados: Se obtuvieron 86 registros de la cacería del oso en 66 poblados de las 16 áreas protegidas evaluadas. El $60.8 \%$ señaló que cazan al oso por considerarlo perjudicial por alimentarse de los cultivos (48.5 \%) y atacar al ganado (42.2\%). Se reportaron 12 cultivos consumidos, siendo el maíz el principal $(n=150,93.3 \%$ ); y cinco especies de ganado, siendo el vacuno el más frecuentemente depredado $(n=131,95.9 \%)$. La frecuencia y años de ataques fue muy variable, realizados mayormente por los machos adultos solitarios. Trece personas aseguraron haber presenciado un total de diez eventos de ataque, dando una descripción detallada, mientras que tres personas comentaron observar comportamientos de carroñeo.

Discusión y conclusiones: El ingreso del oso a los cultivos de maíz es un evento oportunista, debido a que es un alimento con altos niveles de energía y proteínas, y fácil de acceder principalmente en las áreas adyacentes a los bosques premontanos y montanos. Por otro lado, si bien existen investigaciones que confirman la depredación del ganado vacuno en Venezuela, Colombia, Ecuador y Bolivia, en el Perú aún no se ha hecho un estudio al respecto. Sin embargo, 13 personas proporcionaron información muy detallada de sus observaciones, las cuales coincidieron con otras investigaciones en otros países, lo que confirmaría que esta depredación también se da en el Perú. Debido a que ambos hechos son un fuerte motivo de la cacería del oso andino, será importante realizar diversas investigaciones para conocer las causas por los que algunos osos depredan ganado e ingresan a los cultivos. Asimismo, se deberá de trabajar conjuntamente con las comunidades para plantear medidas de mitigación acorde con las condiciones de cada lugar que busquen reducir estas interacciones y por lo tanto la cacería de la especie.

Palabras clave: cacería, conservación, depredación de ganado, consumo de cultivos, mitigación, oso andino

\section{Literatura citada}

Acosta, J. 1849. Viajes Científicos a los Andes Ecuatoriales: Memoria El Tapir Pinchaque por D. Roulin. Librería Castellana, Lasserre. París, Francia.

Albarracín, V. 2010. Percepción actual de los pobladores locales del cantón Lambate sobre el Jucumari (Tremarctos ornatus), La Paz-Bolivia. Tesis de Licenciatura, Universidad Tecnológica Boliviana. La Paz, Bolivia.

Asocebú (Asociación Colombiana de Criadores de Ganado Cebú). 2014. Brahman. [Fecha de acceso Septiembre 2014] <http://www.asocebu.com/Inicio/Comunidad/Razas/Brahman.aspx>.

BaIker, J. 2011. Guía ecoturística: Mancomunidad Saywite-Choquequirao-Ampay (Apurímac, Perú). Con especial referencia a la identificación de fauna, flora, hongos y líquenes en el departamento de Apurímac y sitios adyacentes en el departamento de Cusco. Serie Investigación y Sistematización No 15. Programa Regional Ecobona-Intercooperation. Lima, Perú. 
Bejarano, M. S. 1999. Protección a la vida silvestre: ¿Los buenos juicios prácticos de los campesinos pueden contar? El caso de la zona entre Baeza y Cosanga, provincia del Napo, Ecuador. Facultad Latinoamericana de Ciencias Sociales-Sede Ecuador. Quito, Ecuador.

Butchart, S., R. Barnes, C. Davies, M. Fernández, y N. Seddon. 1995. Threatened mammals of the Cordillera de Colán, Peru. Oryx 29:275-281.

Castellanos, A. 2002. Ataques de oso andino a ganado vacuno en la cuenca del río Cosanga, Ecuador. Ukuku 4(1).

Castellanos, A. 2006. Cannibalism in Andean bears? International Bear News 15:20.

Castellanos, A. 2010. Guía para la rehabilitación, liberación y seguimiento de osos andinos. Andean Bear Foundation. Quito, Ecuador.

Castellanos, A. 2011. Do Andean bears attack mountain tapirs? International Bear News 20:41-42.

Castellanos, A., y A. Laguna. 2012. Depredación a ganado vacuno y mamíferos silvestres por oso andino en el norte de Ecuador. Pp. 112 in Memorias del X Congreso Internacional de Manejo de Fauna Silvestre en la Amazonía y América Latina. Salta, Argentina.

Castellanos, A., M. Altamirano, y G. Tapia. 2005. Ecología y comportamiento de osos andinos reintroducidos en la Reserva Biológica Maquipucuna, Ecuador: implicaciones en la conservación. Revista Politécnica 26:54-82.

Castellanos, A., A. Laguna, y S. Clifford. 2011. Suggestions for mitigating cattle depredation and resulting human-bear conflicts in Ecuador. International Bear News 20:16-18.

Chaunan, N. P. S. 1999. Evaluation of crop damage in the eco-development project area to suggest mitigation measures. Wildlife Institute of India. Dehra Dun, India.

Clarkson, P. L., y I. Stirling. 1994. Polar bears. Pp. C25-C34 in Prevention and control of wildlife damage (Hygnstrom, S. E., R. M. Timm, y G. E. Larson, eds.). University of Nebraska Press. Lincoln, EE.UU.

CCD (Corporación de Conservación y Desarrollo). 1994. Estudio efecto del oso de anteojos sobre los cultivos de maíz en las comunidades de Bermejo, Cuyuja y Sardinas. Presentado al Proyecto Subir. Quito, Ecuador.

Dierenfeld, E. S. 1989. Nutritional considerations in feeding the captive spectacled bear. Pp. 114-130 in Proceedings of the First International Symposium on the Spectacled Bear (Rosenthal, M. A., ed.). Lincoln Park Zoological Gardens. Chicago, EE.UU.

Figueirido, B., P. Palmovist, y J. A. Pérez-Claros. 2009. Ecomorphological correlates of craniodental variation in bears and paleobiological implications for extinct taxa: an approach based on geometric morphometrics. Journal of Zoology 277:70-80.

FigueroA, J. 2005. Habitat overlap between Andean bear and puma in Peru. 16th International Conference on Bear Research and Management. Riva del Garda, Italia.

Figueroa, J. 2008. Cacería del oso andino en el Perú. Centro Iberoamericano de la Biodiversidad, Universidad de Alicante. Alicante, España.

Figueroa, J. 2013. Revisión de la dieta del oso andino Tremarctos ornatus (Carnivora: Ursidae) en América del Sur y nuevos registros para el Perú. Revista del Museo Argentino de Ciencias Naturales 15: $1-27$.

Figueroa, J., y M. Stucchi. 2002. Situación actual del oso andino en el Santuario Histórico de Machu Picchu y zonas adyacentes. Cusco, Perú. Cooperación Técnica Alemana GTZ-Fanpe y Proyecto Oso Andino Perú. Lima, Perú.

FigueroA, J., y M. Stucchi. 2003. Algunos aspectos etológicos del Tremarctos ornatus (Cuvier, 1825) (Carnivora: Ursidae) "oso andino" en cautiverio. Pp. 210-347 in International Studbook for the Andean bear (Tremarctos ornatus) 2000-2002 (Rosenthal, M. A., ed.). Lincoln Park Zoological Gardens. Chicago, EE.UU.

Figueroa, J., y M. Stucchi. 2013. Presencia del oso andino Tremarctos ornatus (Carnivora: Ursidae) en el Corredor de Conservación Vilcabamba-Amboró, sureste del Perú. Therya 4:511-538. 
Figueroa, J., M. Stucchi, y R. Rojas-VeraPinto. 2013. El oso andino (Tremarctos ornatus) como especie clave para la conservación del bosque seco del Marañón (Cajamarca-Amazonas, Perú). Cooperación Alemana (GIZ), Asociación para la Investigación y Conservación de la Biodiversidad (AICB). Lima, Perú.

Flores, S., M. Bustamante, G. Remache, I. Goldstein, y J. Camacho. 2005. Andean bear-cattle conflict: applying the landscape species conservation model. International Bear News 14:28-30.

Fredriksson, G. 2005. Human-sun bear conflicts in East Kalimantan, Indonesian Borneo. Ursus 16:130-137.

Garcilaso de la Vega, I. 1609. Comentarios Reales de los Incas. Centro de Estudios y Difusión de la Cultura Andina Bartolomé de las Casas. Cusco. [Fecha de acceso Agosto 2014] <http://www. bartolomedelascasas.es/paginas/comenta.htm>

Garshelis, D. L., R. S. Sikes, D. E. Andersen, y E. C. Birney. 1999. Landowners' perceptions of crop damage and management practices related to black bears in east-central Minnesota. Ursus 11:219-224.

Goldstein, I. R. 1989. Distribution, habitat use, and diet of spectacled bear (Tremarctos ornatus) in Venezuela. Pp. 2-16 in Proceedings of the First International Symposium on the Spectacled Bear (Rosenthal, M. A., ed.). Lincoln Park Zoological Gardens. Chicago, EE.UU.

Goldstein, I. 1991. Are spectacled bear's tree nests feeding plataforms or resting places? Mammalia 55:433434.

Goldstein, I. 1992. Spectacled bear predation and feeding behavior on livestock in Venezuela. Studies on Neotropical Fauna and Enviroment 27:1-5.

Goldstein, I. 2002. Andean bear-cattle interactions and tree nest use in Bolivia and Venezuela. Ursus 13:369-372.

Goldstein, I., S. Paisley, R. Wallace, J. P. Jorgenson, F. Cuesta, y A. Castellanos. 2006. Andean bear-livestock conflicts: a review. Ursus 17:8-15.

Guamán Poma de Ayala, F. 2004 [1615-1616]. El primer nueva corónica y buen gobierno. Det Kongelige Bibliotek [Fecha de acceso Agosto 2014] <http://www.kb.dk/permalink/2006/ poma/info/es/ foreword.htm>.

Gunther, K. A., y R. A. Renkin. 1990. Grizzly bear predation on elk calves and other fauna of Yellowstone National Park. International Conference on Bear Research and Management 8:329-334

Herrera, A.M., J. Nassar, F. Michelangeli, J. Rodríguez, y D. Torres. 1994. The spectacled bear in the Sierra Nevada National Park of Venezuela. International Conference on Bear Research and Management 9:149-156.

Humboldt von, A. 2004 [1801-1802]. Mi viaje por el camino del Inca (1801-1802): Quito, Cuenca, Cajamarca, Trujillo, Lima. Editorial Universitaria. Santiago de Chile, Chile.

Huygens, O. C., Y H. Hayashı. 1999. Using electric fences to reduce Asiatic black bear depredation in Nagano prefecture, central Japan. Wildlife Society Bulletin 27: 959-964.

Huygens, O. C., F. T. Van Manen, D. A. Martorello, H. Hayashi, y J. Ishida. 2004. Relationships between Asiatic black bear kills and depredation costs in Nagano Prefecture, Japan. Ursus 15: 97-202.

Hwang, M. H., D. L. Garshelis, y Y. Wang. 2002. Diets of Asiatic black bears in Taiwan, with methodological and geographical comparisons. Ursus 13:111-125.

Inocente, O., L. Sumar, y A. LoaizA. 2006. Denominación de origen de maíz blanco gigante. Cosude-Agencia Suiza para el Desarrollo y la Cooperación. Programa Pymagros. Lima, Perú.

Jiménez de LA EspadA, M. 1965 [1582]. Relaciones Geográficas de Indias: Perú. Volumen 184. Tomo II. Biblioteca de Autores Españoles. Madrid, España.

Jonker, S. A., J. A. Parkhurst, R. Field, y T. K. Fuller. 1998. Black bear depredation on agricultural commodities in Massachusetts. Wildlife Society Bulletin 26:318-324.

Jorgensen, C. J. 1979. Bear-sheep interactions, Targhee National Forest. International Conference on Bear Research and Management 5:191-200.

Jorgenson, J. P., y S. Sandoval. 2005. Andean bear management needs and interactions with humans in Colombia. Ursus 16:108-116. 
KarLsson, J., y O. Johansson. 2010. Predictability of repeated carnivore attacks on livestock favours reactive use of mitigation measures. Journal of Applied Ecology 47:166-171.

KLENZENDORF, S. A. 1997. Management of brown bears (Ursus arctos) in Europe. Tesis de Maestría, Virginia Polytechnic Institute and State University. Blacksburg, EE.UU.

Knarrum, V., O. J. Sørensen, T. Eggen, T. Kvam, O. Opseth, K. Overskaug, y A. Eidsmo. 2006. Brown bear predation on domestic sheep in central Norway. Ursus 17:67-74.

Krofel, M., I. Kos, y K. Jerina. 2012. The noble cats and the big bad scavengers: effects of dominant scavengers on solitary predators. Behavioral Ecology and Sociobiology 66:1297-1304.

Laguna, A. 2013. Estudio del conflicto oso andino-humano en los Andes norte de Ecuador. Memorias del II Congreso Ecuatoriano de Mastozoología. Puyo, Ecuador.

Leite Pitman, R. 2008. Leaving passages for wildlife where the Interoceanic Highway will cross the Vilcabamba Amboró corridor. Progressive Report for the Rufford Innovation Award. Cusco, Perú.

Lequanda, DE J. I. 1793a. Continuación de la descripción geográfica de la Ciudad y Partido de Truxillo. Tomo VIII, № 249, folios 52-59. Mercurio Peruano, 23 de mayo de 1793. Lima, Perú.

Lequanda, DE J. I. 1793b. Continuación de la descripción geográfica del Partido de Piura perteneciente á la Intendencia de Truxillo. Tomo VIII, № 264, folios 175-182. Mercurio Peruano, 14 de julio de 1793. Lima, Perú.

Luscomвe, A. 1989. Policy and political consideration in spectacled bear conservation in Peru. Pp. 39-43 in Proceedings of the First International Symposium on the Spectacled Bear (Rosenthal, M. A., ed.). Lincoln Park Zoological Gardens. Chicago, EE.UU.

Morales, A. 2003. Control de daños provocados por fauna silvestre a maizales. Comunidades de Pajan y Sanachi, Municipio Curva, ANMIN Apolobamba, 2001-2003. Wildlife Conservation Society. La Paz, Bolivia.

Murphy, K. M., G. S. Felzien, M. G. Hornocker, y T. K. Ruth. 1998. Encounter competition between bears and cougars: some ecological implications. Ursus 10:55-60

Nallar, R., A. Morales, y H. Gómez. 2008. Manual para la identificación y reconocimiento de eventos de depredación del ganado por carnívoros altoandinos. Wildlife Conservation Society. La Paz, Bolivia.

Normua, F., S. Higashi, L. Ambu, y M. Mohamed. 2004. Notes on oil palm plantation use and seasonal spatial relationships of Sun bears in Sabah, Malaysia. Ursus 15: 227-231.

OJeda, M. C., y A. L. Pesca. 2006. Uso del hábitat natural del oso andino (Tremarctos ornatus) en la Serranía de las Quinchas, Magdalena Medio (Colombia). Tesis de Licenciatura, Universidad Pedagógica y Tecnológica de Colombia, Escuela de Biología. Tunja, Colombia.

Otstavela, T., K. A. Vuoric, D. E. Simsd, A. Valrosa, O. Vainioe, y H. Saloniemia. 2009. The first experience of livestock guarding dogs preventing large carnivore damages in Finland. Estonian Journal of Ecology 58:216-224.

Paisley, S. 2001. Andean bears and people in Apolobamba, Bolivia: Culture, conflicts and conservation. Tesis de Doctorado, Durrell Institute of Conservation and Ecology. University of Kent. Canterbury, Reino Unido.

Payán, C. E. 2004. Managing Carnivores in Colombia's Central Andes. International Bear News 13:21-22.

Peebles, K. A., R. B. Wielgus, B. T. Maletzke, y M. E. Swanson. 2013. Effects of remedial sport hunting on cougar complaints and livestock depredations. PLoS ONE 8(11): e79713. doi:10.1371/journal. pone.0079713.

Peyton, B. 1980. Ecology, distribution and food habits of spectabled bear, Tremarctos ornatus, in Peru. Journal of Mammalogy 61: 639-652.

Peyton, B. 1983. Uso de hábitat por el oso frontino en el Santuario Histórico de Machu Picchu y zonas adyacentes en el Perú. Pp. 23-31 in Simposio Conservación y Manejo Fauna Silvestre Neotropical 
(Aguilar, P. G., ed.). Arequipa, Perú.

Peyton, B. 1987. Habitat components of the spectacled bear in Machu Picchu, Peru. International Conference on Bear Research and Management 7:127-133.

Peyton, B. 1994. Conservation in the developing world: ideas on how to proceed. International Conference on Bear Research and Management 9:115-127.

Peyton, B. 1999. Spectacled bear conservation action plan. Pp. 157-198 in Bears: Status survey and conservation action plan. Compiled by Christopher Servheen, Stephen Herrero y Bernard Peyton. UICN/SSC Bear Specialist Group. Gland, Suiza y Cambridge, Reino Unido.

Peyton, B., E. Yerena, D. Rumiz, J. Jorgenson, y J. Orejuela. 1998. Status of wild Andean bears and policies for their management. Ursus 10:87-100.

PoncE, P. 1991. Relaciones histórico-geográficas de la Audiencia de Quito (siglo XVI-XIX). Tomo II. Consejo Superior de Investigaciones Científicas, Centro de Estudios Históricos, Departamento de Historia de América. Madrid, España.

PovedA, J. J. 1999. Interacciones ganado-oso andino Tremarctos ornatus (F. Cuvier, 1825) en límites de cinco municipios con el Parque Nacional Natural Chingaza: una aproximación cartográfica. Tesis de Licenciatura, Pontificia Universidad Javeriana, Facultad de Ciencias. Santa Fé de Bogotá, Colombia.

Poveda, J. J. s/f. Interacciones ganado oso andino (Tremarctos ornatus) en el límite de cinco municipios con el P.N.N. Chingaza. Una aproximación cartográfica.

RIGG, R. 2001. Livestock guarding dogs: their current use world wide. Occasional Paper 1, Species Survival Commission, International Union for the Conservation of Nature and Natural Resources. Gland, Suiza.

RivadeneiRA, C. 2001. Dispersión de semillas por el oso andino (Tremarctos ornatus) y elementos de su dieta en la región de Apolobamba-Bolivia. Tesis de Licenciatura, Universidad Mayor de San Andrés. La Paz, Bolivia.

Rodríguez, D., C. E. Payán, y M. Barrera. 2004. Regional presence and distribution of the Andean bear (Tremarctos ornatus) and puma (Puma concolor) in strategic conservation ecosystems of the eastern Andean area of Colombia. 15th International Conference on Bear Research and Management. San Diego, EE.UU.

Rodríguez, A., R. Gómez, A. Moreno, C. Cuellar, y D. J. Lizcano. 2014. Record of a mountain tapir attacked by an Andean bear on a camera trap. Tapir Conservation 23:24-25.

Rostworowski, M. 1981. Recursos naturales renovables y pesca, siglos XVI y XVII. Colección Historia Andina 8. Instituto de Estudios Peruanos. Lima, Perú.

Rostworowskı, M. 2005. Ensayos de la historia andina I: élites, etnias, recursos. Instituto de Estudios Peruanos. Lima, Perú.

Rumiz, D., C. Eulert, y R. ARispe. 1999. Situación del oso andino (Tremarctos ornatus) en los Parques Nacionales Amboró y Carrasco, Bolivia. Pp. 375-381 in Manejo y conservación de fauna silvestre en América Latina (Fang, T., O. Montenegro y R. Bodmer, eds.). La Paz, Bolivia.

Solbelzon, L. H. 2002. Los Ursidae (Carnivora, Fissipedia) fósiles de la República Argentina. Aspectos sistemáticos y paleoecológicos. Tesis de Doctorado, Universidad Nacional de la Plata. La Plata, Argentina.

Stephens, D. W., y J. R. KreBs. 1986. Foraging theory (Monographs in behavior and ecology). Princeton University Press. Princeton, EE.UU.

Stucchi, M., y J. Figueroa. 2013. Morfología cráneo-mandibular del oso andino Tremarctos ornatus (Carnivora: Ursidae). Therya 4:485-509.

SUÁREZ, L. 1988. Seasonal distribution and food habits of spectacled bear Tremarctos ornatus in highlands of Ecuador. Studies on Neotropical Fauna and Environment 23:133-136.

ToRREs, D. 2006. Guía para la identificación de señales de presencia del oso frontino (Tremarctos ornatus) en los Andes Venezolanos. Fundación Andígena. Mérida, Venezuela.

Torres, D., A. Lobo, R. Ascanio, y G. Lobo. 1995. Monitoring the spectacled bear (Tremarctos ornatus) populations in the watershed of the Capaz River, Merida State, Venezuela. Sociedad de Ciencias 
Naturales La Salle (Tomo LV) 143:25-40.

Treves, A. 2009. Hunting for large carnivore conservation. Journal of Applied Ecology 46:1350-1356.

Treves, A., K. J. Kapp, y D. M. MacFarLand. 2010. American black bear nuisance complaints and hunter take. Ursus 21:30-42.

Treves, A., L. Naughton Treves, R. A. Rose, E. K. Harper, D. J. Mladenoff, T. A. Sickley, y A. P. Wydeven. 2004. Predicting human-carnivore conflict: a spatial model derived from 25 years of data on wolf predation on livestock. Conservation Biology 18:114-125.

Tschudi, J. J. von. 2003 [1838-1842]. El Perú. Esbozos de viajes realizados entre 1838 y 1842 . Pontificia Universidad Católica del Perú. Lima, Perú.

URBIGKIT, C., Y J. URBIGKIT. 2010. A review: the use of livestock protection dogs in association with large carnivores in the Rocky Mountains. Sheep and Goat Research Journal 25: 1-8.

Van Horn, R., R. Appleton, y J. Amanzo. 2014. Andean bears in two peruvian forests are rarely photographed with meat. International Bear News 23:20-22.

Vargas, J. A., y A. Hernández. 2001. Distribución altitudinal de la mastofauna en la Reserva de Biósfera "El Cielo", Tamaulipas, México. Acta Zoológica Mexicana 82:83-109.

VELA, H. 2009. Meat consumption and use of parts of the Andean bear (Tremarctos ornatus) in the district of Chisquilla and Jumbilla, Bong County, Amazonas-Peru. International Bear News 18:20-21.

Vineyard, T. G., y D. Torres. 2004. Solar electric fencing to prevent Andean bear-human conflicts in the Venezuelan Andes. International Bear News 13:26.

Wıck, P. 1995. Minimising bear-sheep conflicts through herding techniques. International Conference on Bear Research and Management 9:367-373.

Wielgus, R. B., y F. L. Bunnell. 2000. Possible negative effects of adult male mortality on female grizzly bear reproduction. Biological Conservation 93:145-154.

Wielgus, R. B., F. Sarrazin, R. Ferriere, y J. Clobert. 2001. Estimating effects of adult male mortality on grizzly bear population growth and persistence using matrix models. Biological Conservation 98:293-303.

Witmer, G. W., y D. G. WhittakeR. 2001. Dealing with nuisance and depredating black bears. Western Black Bear Workshop 7:73-81.

Yañez, M. A., y C. F. Eulert. 1996. Estudio del estatus actual del oso andino (Tremarctos ornatus Cuvier), en la Serranía de Los Milagros, Prov. Hernando Siles del Dpto. de Chuquisaca (Bolivia). Instituto Científico Alex Pacha. La Paz, Bolivia.

Ziegltrum, G. J., y D. L. Nolte. 1996. Black bear damage management in Washington State. Seventh Eastern Wildlife Damage Management Conference 7:104-107.

Zimmerman, B., P. Wabbakken, y M. Dotterer. 2003. Brown bear-livestock conflicts in a bear conservation zone in Norway: are cattle a good alternative to sheep? Ursus 14:72-83.

Summited: November 5, 2014

Review: January 15, 2015

Accepted: January 27, 2015

Associated editor: Cristina Macswiney 